https://helda.helsinki.fi

\title{
In search of the visual pigment template
}

\section{Govardovskii, V.I.}

Cambridge University Press

2000

Visual Neuroscience. 2000. 17: 509-528

http://hdl.handle.net/1975/950

http://dx.doi.org/doi:10.1017/S0952523800174036

Downloaded from Helda, University of Helsinki institutional repository.

This is an electronic reprint of the original article.

This reprint may differ from the original in pagination and typographic detail.

Please cite the original version. 


\title{
In search of the visual pigment template
}

\author{
VICTOR I. GOVARDOVSKII, ${ }^{1} *$ NANNA FYHRQUIST, ${ }^{2,3}$ TOM REUTER, ${ }^{3}$ DMITRY G. KUZMIN, \\ AND KRISTIAN DONNER ${ }^{2}$ \\ 'Institute of Evolutionary Physiology and Biochemistry, Russian Academy of Sciences, 194223 St. Petersburg, Russia \\ ${ }^{2}$ Department of Biosciences, FIN-00014 University of Helsinki, Finland \\ ${ }^{3}$ Department of Ecology and Systematics, FIN-00014 University of Helsinki, Finland
}

(RECEIVED July 19, 1999; ACCePTED February 2, 2000)

\begin{abstract}
Absorbance spectra were recorded by microspectrophotometry from 39 different rod and cone types representing amphibians, reptiles, and fishes, with A1- or A2-based visual pigments and $\lambda_{\max }$ ranging from 357 to $620 \mathrm{~nm}$. The purpose was to investigate accuracy limits of putative universal templates for visual pigment absorbance spectra, and if possible to amend the templates to overcome the limitations. It was found that (1) the absorbance spectrum of frog rhodopsin extract very precisely parallels that of rod outer segments from the same individual, with only a slight hypsochromic shift in $\lambda_{\max }$, hence templates based on extracts are valid for absorbance in situ; (2) a template based on the bovine rhodopsin extract data of Partridge and De Grip (1991) describes the absorbance of amphibian rod outer segments excellently, contrary to recent electrophysiological results; (3) the $\lambda_{\max } / \lambda$ invariance of spectral shape fails for A1 pigments with small $\lambda_{\max }$ and for A2 pigments with large $\lambda_{\max }$, but the deviations are systematic and can be readily incorporated into, for example, the Lamb (1995) template. We thus propose modified templates for the main " $\alpha$-band" of A1 and A2 pigments and show that these describe both absorbance and spectral sensitivities of photoreceptors over the whole range of $\lambda_{\max }$. Subtraction of the $\alpha$-band from the full absorbance spectrum leaves a " $\beta$-band" described by a $\lambda_{\max }$-dependent Gaussian. We conclude that the idea of universal templates (one for A1 - and one for A2-based visual pigments) remains valid and useful at the present level of accuracy of data on photoreceptor absorbance and sensitivity. The sum of our expressions for the $\alpha$ - and $\beta$-band gives a good description for visual pigment spectra with $\lambda_{\max }>350 \mathrm{~nm}$.
\end{abstract}

Keywords: Retina, Photoreceptors, Rhodopsin, Absorbance, Spectral sensitivity

\section{Introduction}

The idea that the absorbance spectra of all visual pigments can be described by a common template or "nomogram" (Dartnall, 1953) builds on the assumption that all curves have the same basic shape, subject only to a rule of transformation to different wavelengths of maximum absorbance $\left(\lambda_{\max }\right)$. The spectrum of any pigment can then be completely characterized with the single parameter $\lambda_{\max }$. To date, no comprehensive physical theory exists for the relation between molecular structure and absorbance characteristics, and the very notion of a universally valid template, as well as the specifics of its design, depend wholly on empirical curve-fitting to recorded data. The considerable effort devoted to refining templates after Dartnall's pioneering work has been motivated mainly by their great practical value in the treatment and interpretation of all kinds of spectral data, but also by the prospect that good tem-

Address correspondence and reprint requests to: Victor I. Govardovskii, Institute of Evolutionary Physiology and Biochemistry, Russian Academy of Sciences, Thorez Prospect 44, 194223 St. Petersburg, Russia. E-mail: vgov@mailbox.alkor.ru

*Until September 2000 at: Howe Laboratories, Harvard Medical School, MEEI, 243 Charles St., Boston, MA 02114, USA. E-mail: vgovardovski@ yahoo.com plates may ultimately shed light on the underlying physics (Bridges, 1967; Munz \& Schwanzara, 1967; Greenberg et al., 1975; Hárosi, 1976; Knowles \& Dartnall, 1977; Ebrey \& Honig, 1977; Metzler \& Harris, 1978; Dawis, 1981; Barlow, 1982; Mooij \& van den Berg, 1983; Baylor et al., 1984, 1987; MacNichol, 1986; Mansfield et al., 1986; Maximov, 1988; Makino et al., 1990; Partridge \& De Grip, 1991; Kraft et al., 1993; Stavenga et al., 1993; Hárosi, 1994; Lamb, 1995; Makino \& Dodd, 1996; Palacios et al., 1996, $1998 a, b)$.

During the last decades, however, attempts to create a template have been largely limited to devising various mathematical expressions that would describe a particular set of spectral data. Three basic experimental questions related to template design have not been satisfactorily answered:

1. Is it really true that the shape of the spectral absorbance curve is uniquely defined by $\lambda_{\max }$ ? In other words, to what level of accuracy is it true that visual pigments having the same $\lambda_{\max }$, but coming from different species or photoreceptor types (rods vs. cones) will have congruent absorbance spectra? This question, central to the very idea of templates, has received little systematic attention after Dartnall. Recent results by Palacios et al. (1998a) indicate significant discrep- 
ancies between sensitivity spectra from single frog rods, and mammalian or fish rhodopsin templates. Addressing it requires high-quality spectra with similar $\lambda_{\max }$ from different sources. In the present paper, we compare new "optimized" recordings of the absorbance of toad and frog rod outer segments (OSs) with the best available mammalian template.

2. What is the form of the standard curve? If the idea of a universal template holds, this is a straightforward question, only requiring good measurements on any conveniently available pigment, irrespective to its origin and $\lambda_{\max }$. There has been considerable technical progress since Dartnall (1953), and today the absorbance spectrum of bovine rhodopsin measured by Partridge and De Grip (1991) from highly purified extracts provides a curve of reference quality for A1 pigments. Among A2 pigments, the spectrum recorded from (somewhat less rigorously purified) carp porphyropsin extract by Bridges (1967) offers an acceptable standard. However, these standards are from pigments in solution and there remains the question how well they represent the absorbance of the pigment in situ in the OS. We study this by measuring, in the same microspectrophotometer, the absorbances of extracts and intact OSs prepared from the same individual frog retinas,

3. Once a standard exists, how should it be transformed as function of $\lambda_{\max }$ ? A rule that had already gained wide acceptance, that spectra are shape invariant on a $\lambda_{\max } / \lambda$ scale (Mansfield, 1985; MacNichol, 1986; Mansfield et al., 1986), has recently been shown to fail for $\mathrm{Al}$ pigments peaking at short wavelengths (Hárosi, 1994), as is true also of A2 pigments peaking at long wavelengths (see Fig. 8 of the present work). In the present work, we recorded spectra from a number of pigments with $\lambda_{\max }$ spaced over a wide range to determine how the $\lambda_{\max } / \lambda$ invariance, in the following referred to as the MM (Mansfield-MacNichol) transform, might be modified to produce a template that applies over the whole known $\lambda_{\max }$ range.

We report new microspectrophotometry (MSP) data on the absorbance of 39 visual pigments in situ in OSs. In obtaining the data, we paid particular attention to a high signal-to-noise ratio (SNR) and elimination of distorting factors (cf. Appendices 1 and 2). To cover extreme spectral positions, mainly occupied by cones, and yet measure from reasonably large OSs, we focused on amphibian, gecko, and fish photoreceptors.

Comparing the bovine Al standard with MSP data from rhodopsin rods of several amphibian species, we find no support for the notion that visual pigments with nearly the same $\lambda_{\max }$ may have absorbance spectra of significantly different shape. Thus, the idea of a universal template remains valid on this central point. We further find excellent agreement between the absorbance spectrum of frog rhodopsin in solution and that of intact rod OSs from the same retina, save for a small $(c a .1 \mathrm{~nm})$ shift in $\lambda_{\max }$ consistent with "Kundt's rule"(i.e. the shift of an absorbance spectrum to longer wavelengths in more refractive media). This justifies the use of standards based on extracts, at least for rod pigments.

In the vertebrate groups studied, the absorbance spectra of A1 rod and cone pigments with $\lambda_{\max }>480 \mathrm{~nm}$ are well predicted by a template based on the bovine standard plus the MM transform. For A2 pigments, MM transforms of the carp standard are good for $\lambda_{\max }$ between $c a .450 \mathrm{~nm}$ and $540 \mathrm{~nm}$. Increasing discrepancies are observed in A1 pigments with smaller $\lambda_{\max }$ and $\mathrm{A} 2$ pigments with larger $\lambda_{\max }$; in both cases long-wavelength absorbance decays more steeply than predicted. We propose expressions (separately for A1 and A2) that modify the MM transform as incorporated into Lamb's (1995) formula so that it provides a good description of the main absorbance band (the $\alpha$-band) over the whole $\lambda_{\max }$ range. We then decompose spectra into $\alpha$ - and $\beta$-bands by subtraction of the $\alpha$-band template from the complete experimental spectra. The $\beta$-bands thus isolated were well described by $\lambda_{\max }$-dependent Gaussians. The sum of our $\alpha$ - and $\beta$-band template provides a complete description of presently known absorbance spectra with $\lambda_{\max }>$ $350 \mathrm{~nm}$.

\section{Methods}

\section{Animals}

Measurements were made on rods and cones of several species of fish (sturgeons Acipenser güldenstädti, and A. stellatus; hybrid A. baeri $x$ A. medirostris; carp Cyprinus carpio; golfish Carassius auratus; giant danio Danio aequipinnatus; zebrafish Brachydanio rerio), frogs (Rana temporaria, $R$. ridibunda, $R$. catesbeiana, and $R$. pipiens), toads (Bufo bufo and B. marinus), clawed toad (Xenopus laevis), and geckos (Gymnodactylus caspius and Hemidactylus sp., presumably $H$. frenatus). The common toad (B. bufo) and the frog species except $R$. catesbeiana were kept unfed in hibernating conditions at about $4^{\circ} \mathrm{C}$ for up to a month. Before the experiment, they were brought for a day to room temperature. Other animals were kept at room temperature and 12-h light/12-h dark regime, and supplied with appropriate food.

\section{Preparation}

Prior to the experiment, animals were dark adapted overnight. They were decapitated and double-pithed and the eyes were removed under weak red light $(\lambda>680 \mathrm{~nm})$. When working with long-wave $\left(\lambda_{\max }>500 \mathrm{~nm}\right)$ visual pigments, all further preparation was done under infrared light with an infrared viewer (FindR-Scope, FJW Industries, IL). For short-wave pigments $\left(\lambda_{\max }<\right.$ $500 \mathrm{~nm})$, deep red light $(\lambda>640 \mathrm{~nm})$ was sometimes preferred, as it facilitated manipulations without causing perceptible bleach. Retinas were isolated in physiological saline containing for fish and amphibia (mM): $\mathrm{NaCl}, 110 ; \mathrm{KCl}, 2.5 ; \mathrm{CaCl}_{2}, 1 ; \mathrm{MgSO}_{4}, 1$; $\mathrm{NaHCO}_{3}, 10$; glucose, 10 . The solution was buffered to $\mathrm{pH} 7.2-7.5$ with $10 \mathrm{mM}$ HEPES. For gecko retinas, $[\mathrm{NaCl}]$ was increased to $140 \mathrm{mM}$. In some cases, $10-15 \%$ dextran (Mol. wt. $=70 \mathrm{kDa})$ or $0.2 \%$ methylcellulose was added to prevent cell movement. When indicated in the text, $10-20 \mathrm{mM}$ of freshly neutralized hydroxylamine was added to the dissection saline.

Pieces of retina separated from the pigment epithelium were transferred to a drop of the same saline on a coverslip and teased apart. The sample was covered with a second slip, sealed at the edges with vaseline, and placed on the MSP stage.

\section{Microspectrophotometry}

MSP was performed with two basically similar instruments, built in St. Petersburg and Helsinki. They are straightforward singlebeam, computer-controlled devices, where the photomultiplier output (Hamamatsu R928 tube) is fed to the computer memory via a current-to-voltage transducer and a 12-bit A/D converter. A halogen lamp served as a light source both for the visible and near-UV (down to $330 \mathrm{~nm}$ ) region. Spectral lights were obtained with a grating monochromator controlled by a computer-driven stepper 
motor. Both the instruments are supplied with computer-controlled neutral density filters (O.D. approximately 1 ) that were put in the light path when scanning above $420 \mathrm{~nm}$ and removed at shorter wavelengths, significantly improving performance in the near UV.

An adjustable beam mask was placed at the monochromator's exit slit and imaged (with demagnification) in the sample plane by a quartz-mirror condenser (Schwartzschield system): a 40×, NA 0.5 , long-working distance ( $c a .6 \mathrm{~mm}$ ) objective from the Leningrad Optical-Mechanical Company (LOMC, Russia). These objectives made in the 1960 s are excellently corrected with respect to both axial and lateral chromatic aberrations and can be used at least between 300 and $800 \mathrm{~nm}$. The aperture was normally limited with an extra stop to approximately 0.4 . For collecting and viewing, we used a standard dry $40 \times$, NA 0.95 microscope objective (LOMC) giving a large visual field, yet a reasonably high NA. The combination of a relatively low illumination aperture and high collecting aperture is advantageous in reducing effects of light scattering, diffraction, and refraction (Hárosi, 1975a, Levine \& MacNichol, 1985). We could use this condenser/objective combination down to $350 \mathrm{~nm}$, where light attenuation by the glass objective began to cause significantly increased noise. A UV objective $(58 \times$, NA 0.65$)$ was sometimes used to improve performance in this range. It enabled measurement down to $330 \mathrm{~nm}$, but was associated with increased scatter problems.

Recordings were made either on isolated OSs, isolated cells, or cells attached to the retina. The measuring beam was polarized with the $e$-vector in the plane of the OS disks (i.e. perpendicular to the long axis of the cell). Beam dimensions varied depending on the sample. To minimize problems from refraction and reflection at the outer segment border, the width was normally $1 / 5$ to $1 / 4$ of the OS diameter. With smaller cones (e. g. in Danio red-sensitive cells), it was increased to $c a .1 / 3$ of the OS at its base.

The only substantial difference between the two instruments is the speed of the spectral scan, $33 \mathrm{~nm} / \mathrm{s}$ in Helsinki, and $160 \mathrm{~nm} / \mathrm{s}$ in St. Petersburg. Light intensity data were collected at each step of the monochromator-driving motor, six data points/nm in Helsinki and four data points/nm in St. Petersburg. Data for each $1-\mathrm{nm}$ interval were pooled and stored in the computer memory, separately for downward (red to blue) and upward (blue to red) scans. Scans always started from the longest wavelength, which, depending on the task, varied from 790 to $550 \mathrm{~nm}$. For baseline recording, the measuring beam was first positioned in a clear space near the cell selected for study, and four scans in each direction were averaged. Then the OS was moved into the beam and scanned once in both directions. Optical densities were calculated against corresponding baselines and displayed on the screen separately for each of the two scan directions; thus any substantial difference, indicative of strong bleaching or cell movement, could be readily detected. Usually, the optical densities were finally computed as the means of the values from opposite scan directions.

The wavelength calibration was checked regularly with a mercury lamp. The stability of the calibration and the agreement between the two MSP instruments was confirmed on each experimental day by recording the spectrum of a "blue glass" standard, whose absorbance had also been measured with two standard spectrophotometers (Hitachi 150-20 in St. Petersburg and Hitachi-2000 in Helsinki). Plotting the difference between the recordings provided a sensitive tool for detecting and quantifying possible changes. Day-to-day reproducibility was within $c a .0 .2 \mathrm{~nm}$ in Helsinki and $0.4 \mathrm{~nm}$ in St. Petersburg. IR viewers (Find-R-Scope, FJW Industries) were used for sample observation and adjustments; in St. Petersburg a closed-circuit IR-TV system was also available.

\section{Visual pigment extraction}

To compare rod OS absorbance with the rhodopsin spectrum in solution, digitonin extracts of Rana ridibunda retinas were prepared basically as described by Reuter et al. (1971). Briefly, retinas were detached from pigment epithelium, bathed in frog saline, $\mathrm{pH} 8.0$ (phosphate) and $\mathrm{pH} 4.6$ (acetate) buffers, and rinsed in distilled water. They were centrifuged at $10,000 \mathrm{~g}$ for $15 \mathrm{~min}$ and the water was decanted and replaced with $0.3-1.0 \mathrm{ml}$ of $2 \%$ digitonin (a 1960s stock of Merck digitonin from George Wald's laboratory) in $125 \mathrm{mM} \mathrm{pH} 6.5$ phosphate buffer. After stirring, the preparation was left for $3 \mathrm{~h}$ at room temperature. Freshly neutralized hydroxylamine $\left(\mathrm{NH}_{2} \mathrm{OH}\right)$ solution was added to a final concentration of 10 or $20 \mathrm{mM}$. The sample was stirred, centrifuged at $50,000 \mathrm{~g}$ for $30 \mathrm{~min}$, and the supernatant extract of visual pigment was pipetted off.

\section{Data processing}

Data processing included averaging, zero line correction, filtering, and template fitting. Most of the procedures varied depending on the sample, as explained in the appropriate places. Here we describe only filtering and template-fitting procedures.

\section{Digital filtering}

High-frequency noise components were removed by Fourier filtering using FFT (Fast Fourier Transform). The normal data array of 300-400 data points is not appropriate for the FFT algorithm and was therefore extended to 512 points, filling in with a cosine transition between the levels at the end of the spectrum's long-wave tail and the start of the $\beta$-band. The transition points were computed as $D_{l}+\left(D_{s}-D_{l}\right)[1-\cos (\pi n /(m+1))] / 2$, where $D_{l}$ and $D_{s}$ are the averages of the 20 last points in the long- and short-wave ends, respectively, $m$ is the total number of points to be filled in, and $n$ is the number of the point in question. This provided a relatively smooth periodical function on which FFT was performed. A certain number of higher harmonics was cut off with a rectangular window, and the smoothed spectrum was obtained by reverse FFT. Computer simulations with noiseless model curves resembling visual pigment spectra show that if 25 harmonics are retained in a spectrum $300 \mathrm{~nm}$ long, the restored curve coincides with the original spectrum to within a fraction of a percent. Normally we used less heavy filtering, retaining 30-35 harmonics. Sometimes we used filtering by convolution with a Gaussian with standard deviation (SD) in the range $1-5 \mathrm{~nm}$.

\section{Template fitting to the experimental spectra}

A first guess $\lambda_{\max }$ for fitting was provided by an operator picking an apparent "true" peak among the noise fluctuations. The peak absorbance was computed as the mean of 21 points centered on this guess, divided by 0.9896 , the correction factor derived from a rhodopsin template. Then, as suggested by MacNichol (1986), the long-wave limb between 70 and $30 \%$ of maximum absorbance was fitted with a least-square straight line and the wavelength corresponding to $50 \%$ of maximum absorbance $\left(\lambda_{0.5}\right)$ determined from the line. Assuming that the MM transform is valid, $\lambda_{\max }=$ $\lambda_{0.5} / k$, where we used $k=1.0925$ for $\mathrm{Al}$ pigments. The slight difference between this value derived from bovine data (Partridge \& De Grip, 1991) and that given by MacNichol (1986) (1.0924, based on Dartnall's frog data) does not produce significant differences in estimated $\lambda_{\max }$. For A2 pigments, we used $k=1.1121$, derived from Bridges' (1967) carp data. Compared with the value 
1.1101 obtained by MacNichol (1986) from the salmon data of Munz and Schwanzara (1967), our value blue-shifts the estimated $\lambda_{\max }$ by $\mathrm{ca}$. $1 \mathrm{~nm}$. The data-processing program contains a set of standard templates based on different data sets on the absorbance spectra of frog ( $R$. temporaria, Knowles \& Dartnall, 1977; $R$. pipiens, Donner \& Reuter, 1976) and bovine (Partridge \& De Grip, 1991) rhodopsins and carp porphyropsin (Bridges, 1967). Any of these curves could be MM transformed to the estimated $\lambda_{\max }$ and superimposed on the experimental data. Mixed A1/A2 spectra could also be generated, whereby the $\lambda_{\max }$ difference within an $\mathrm{A} 1 / \mathrm{A} 2$ pair was determined either from the Dartnall and Lythgoe (1965), Whitmore and Bowmaker (1989), or the Hárosi (1994) relation.

\section{Results}

\section{Amphibian rods: A standard}

The large size of amphibian rod OSs makes them suitable $a$ priori for generating MSP data of optimal quality. This is a prerequisite for answering two of our main questions (cf. Introduction):

1. How well does the bovine standard of Partridge and De Grip (1991) apply to other Al pigments with similar $\lambda_{\max }$ in general, and to amphibian rods in particular (cf. Palacios et al., 1998a)?

2. How closely does the absorbance of a visual pigment in detergent solution match that of the same pigment in situ in photoreceptors?

Optimization of MSP recordings is essentially a matter of balance between two opposing pressures: that of using a highintensity measuring beam to maximize SNR and that of using as little light as possible to minimize distortion of the spectrum due to bleaching. Our solution is described in detail in Appendix 1. Briefly, we accept that bleaching must occur, but we measure its effect on the shape of the spectrum and correct for it.

Fig. 1 gives an idea of the general quality of MSP data that can be obtained from amphibian rods. The spectrum, shown on both linear (A) and logarithmic ordinates (B, after subtracting the zero line), is the average of recordings from 20 rods of Bufo bufo with an intensity resulting in $\mathrm{ca}$. $2 \%$ bleach. The individual spectra were normalized at $502 \mathrm{~nm}$ prior to averaging, thus eliminating data scatter due to variations in OS diameters and pigment densities. The average curve and SD have then been rescaled to the mean peak absorbance in the same sample. As seen in panel A, the zero absorbance line for these big OSs is virtually horizontal, and the zero offset, $3.7 \times 10^{-3} \mathrm{OD}$, is only slightly above that expected from two reflections at the $\mathrm{OS} /$ medium interface. As seen in panel $\mathrm{B}$, the absorbance values are usable down to about 0.002 of maximum (reached around $625 \mathrm{~nm}$ ). Panel $\mathrm{C}$ shows the spectrum after correction for bleaching as described in Appendix $\mathrm{I}$ and Fourier filtering leaving 35 harmonics (smooth line).

\section{Bovine vs. amphibian rhodopsin}

In Fig. 1C, the corrected absorbance is compared with the bovine template $\mathrm{MM}$ transformed to $\lambda_{\max }=501.4$ (dots). Obviously, the agreement is good over most of the spectral range. The incipient divergence near $400 \mathrm{~nm}$ may be due at least in part to underestimation of the bleach correction (see Appendix 1).
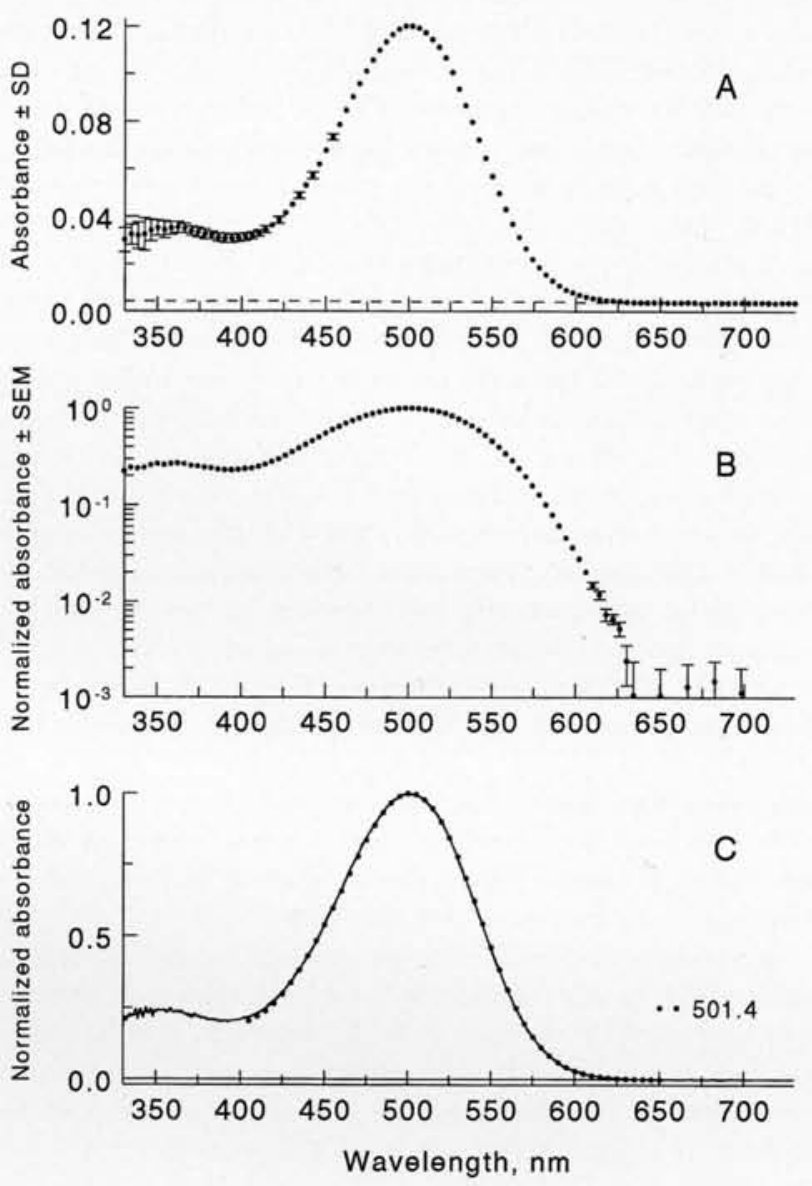

Fig. 1. Absorbance spectra recorded by MSP from rhodopsin rods of Bufo bufo. (A) Averaged spectra of 20 outer segments, normalized prior to averaging at $502 \mathrm{~nm}$. No smoothing applied. The zero (dashed) line is the extrapolation of the least-square fit to the points between 650 and $750 \mathrm{~nm}$. The error bars give SD. (B) The same data plotted on logarithmic ordinates after zero-line subtraction (slope $-1.7 \times 10^{-6} \mathrm{~nm}^{-1}$, zero offset $3.66 \times$ $10^{-3}$ at $750 \mathrm{~nm}$ ). The errors bars give SEM. (C) The noisy curve shows the final absorbance spectrum corrected for bleaching as explained in Appendix 1. The smooth curve is the same spectrum after Fourier filtering with 35 harmonics retained. The dots show the template based on bovine rhodopsin (Partridge \& De Grip, 1991) MM transformed to $\lambda_{\max }=501.4 \mathrm{~nm}$.

An equally good agreement with the MM-transformed bovine template was found for rhodopsin rods in the other amphibians studied (Bufo marinus, Rana temporaria, $R$. ridibunda, and $R$. catesbeiana). There was virtually no variation in $\lambda_{\max }$ among individual rods from the same retina. Values from good-quality spectra, obtained by automated fitting based on the right limb of the curve (see Methods), coincided within $0.3 \mathrm{~nm}$. The mean $\lambda_{\max }$ values for best template fits were $501.7 \mathrm{~nm}$ (range: 501.4-502.5, five animals) in Bufo bufo, $503.3 \mathrm{~nm}$ (range: 503.2-503.4, five animals) in Bufo marinus, $502.1 \mathrm{~nm}$ (range: 501.8-502.5, eight animals) in Rana ridibunda, and $501.6 \mathrm{~nm}$ (two animals) in $R$. catesbeiana. Thus, variations between individuals in these four species did not exceed $1 \mathrm{~nm}$. In $R$. temporaria, however, estimated $\lambda_{\max }$ varied over a 2-nm range in 12 animals, from 502.3 to $504.4 \mathrm{~nm}$ (mean 503.4). Intraspecific variability in $R$. temporaria has previously been reported by Bowmaker et al. (1975). We also made recordings from two $R$. pipiens, but this was before we had devel- 
oped the method for bleach correction. The spectra (not shown) were wider than the bovine template in the short-wave end, most probably due to the accumulation of bleaching products. This does not affect the determination of $\lambda_{\max }$ from the long-wave limb, though, and we obtained $\lambda_{\max }=502.4$ and $502.7 \mathrm{~nm}$ in the two animals.

The excellent fit of the bovine template to toad rhodopsins cannot be a result of fortuitous broadening of a narrower $\mathrm{Al}$ spectrum through admixture of A2 chromophore, as the genus Bufo does not possess A2 even in the tadpole stage (Crescitelli, 1958; Muntz \& Reuter, 1966; cf. also Partridge et al., 1992). By contrast, the Rana species use (mainly or partly) the A2 chromophore in the tadpole stage (Wald, 1946; Liebman \& Entine, 1968; Reuter, 1969). $R$. catesbeiana retains an A2 field of variable size in the dorsal retina throughout its life (Reuter et al., 1971). It has been suggested that other Rana species might also retain a small proportion of A2 even in the adult stage (e.g. Liebman \& Entine, 1968). The bullfrogs we used had been kept in conditions favoring low A2 content (temperature above $20^{\circ} \mathrm{C}$, low light, Tsin \& Beatty, 1980), and the recordings were done on rods from the ventral (A1) part of the eye (cf. Donner et al., 1990). Our technique would detect as little as $2 \%$ of visual pigment having the A2 chromophore. However, no frog rod in our samples showed higher absorbance than the bovine template in the long-wave end, so as to suggest the presence of $\mathrm{A} 2$.

\section{Rhodopsin extracts vs. intact rod outer segments}

Spectra in solution are generally thought to differ from those in situ, as expressed by "Kundt's rule" that predicts a long-wave shift in more refractive medium (see e.g. Bowmaker, 1972, 1973). In Fig. 2A, the Bufo bufo spectrum from Fig. 1C is compared with the template of Knowles and Dartnall (1977), based on extract of frog rhodopsin (dots). Indeed, the fit is not quite good, as the extract template runs systematically above the MSP data in the long-wave end. Similar discrepancy between "Dartnall nomogram" and absorbance spectra of single outer segments is seen in other goodquality recordings from large outer segments of, for example, geckos (Crescitelli et al., 1977).

To study this question in a manner that would exclude extra, "irrelevant" variability as far as possible, we recorded MSP spectra and digitonin extract spectra (ESP) with the same instrument in samples prepared from the same individual. For the ESP, a flat 0.4-ml, 8 -mm light path cuvette was placed into the near-parallel beam between the beam mask and condenser of our MSP instruments. A cuvette filled with pure digitonin solution was used as reference. We used Rana ridibunda, because its large eyes yielded enough material for two to three ESP samples from the same individual.

The dark spectra of extracts and OSs could not be compared directly, as the short-wave absorbance of our extracts was increased by light scattering and impurities. Instead, hydroxylamine postbleach difference spectra were normalized at their maxima and compared (Fig. 2B). From $465 \mathrm{~nm}$ upwards, all points of the extract spectrum (dots) are seen to be blue-shifted compared with the $\mathrm{OS}$ recording (continuous curve). Below $465 \mathrm{~nm}$, comparisons are misleading owing to different contributions of retinal oxime to the difference spectra in extracts and OSs. In the OS, the absorbing dipole of retinal oxime is preferentially orientated across the disk plane (Hárosi \& MacNichol, 1974b), so its absorbance under illumination polarized orthogonally to this direction is much less prominent than in solution, where its orientation is random.
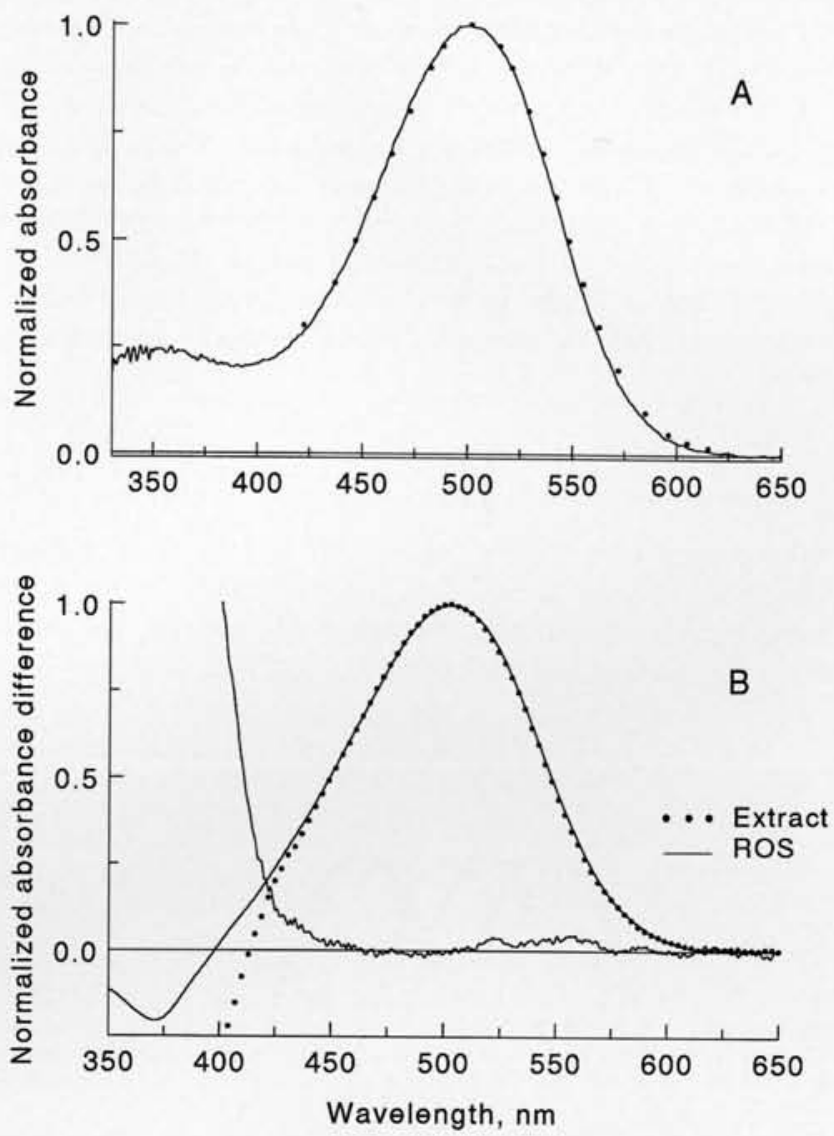

Fig. 2. Comparison between rhodopsin absorbances in detergent extracts and in intact OS. (A) Continuous curve: Bufo bufo rhodopsin spectrum reproduced from Fig. 1C, dots: the Knowles and Dartnall (1977) template based on frog rhodopsin extract. (B) Hydroxylamine postbleach difference spectra of rhodopsin extract (dots) and MSP (average of 21 OSs, corrected for bleaching and Fourier filtered with 35 harmonics retained) from rods of Rana ridibunda. The extracts and isolated cells were obtained from the same animal. The noisy curve shows the difference between extract and OS, multiplied by the factor 3 for clarity. The height of its peak at $550 \mathrm{~nm}$ indicates that the extract is shifted by $-0.8 \mathrm{~nm}$ compared with the OS spectrum.

The difference between MSP and ESP (noisy line in Fig. 2B) is a sensitive measure of the spectral shift. The amplitude of its peak at $550 \mathrm{~nm}$ translates into a $0.8-\mathrm{nm}$ displacement. A similar shift was consistently observed in all five $R$. ridibunda studied, both with the Helsinki and the St. Petersburg instrument. Recordings of our standard blue glass (see Methods) during the same experimental day did not indicate perceptible variability in wavelength calibration. Thus, the hypsochromic shift of the spectrum in digitonin extract compared with the photoreceptor membrane, however small, seems real. Its mean magnitude was $1.0 \mathrm{~nm}$ (range: $0.8-1.5 \mathrm{~nm}$ ).

We also tested the possibility that rhodopsin absorbance in the OS can be influenced by $\mathrm{pH}$ or the ionic milieu of the photoreceptor membrane, as suggested by Bowmaker (1973). Prior to preparing MSP samples, retinas were incubated for up to $24 \mathrm{~h}$ at $4^{\circ} \mathrm{C}$ in saline with pH adjusted to either 6.0, 7.5 (Na-phosphate buffer), or 9.0 (Tris). Samples were sealed in the same saline. Even in intact rods, intracellular $\mathrm{pH}$ partly follows extracellular $\mathrm{pH}$ changes (Saarikoski et al., 1997), and our measurements were done on isolated OSs, which are expected not to regulate $\mathrm{pH}$ at all. Small 
$\mathrm{pH}$ effects could be found in raw spectra, particularly in the shortwave limb. They disappeared after correction for bleaching (data not shown), implicating the $\mathrm{pH}$ dependence of the interconversion of bleaching products. Similarly, no consistent effect $(>1 \%)$ was found when all chloride in the solution was replaced by $\mathrm{NO}_{3}^{-}$, which is known to produce a powerful hypsochromic shift in longwave cone pigments whose opsins have a specific chloride-binding pocket (Fager \& Fager, 1979; Crescitelli, 1980, 1981; Govardovskii et al., 1985; Novitskii et al., 1989; Kleinschmidt \& Hárosi, 1992).

\section{Al pigments with widely differing $\lambda_{\max }$}

To investigate how closely spectra with $\lambda_{\max }$ far from $500 \mathrm{~nm}$ agree with MM transforms of the bovine template, we recorded from amphibian "green" rods, transmuted rods in geckos, and cones in a fish, Danio aequipinnatus (Fig. 3). The data from relatively
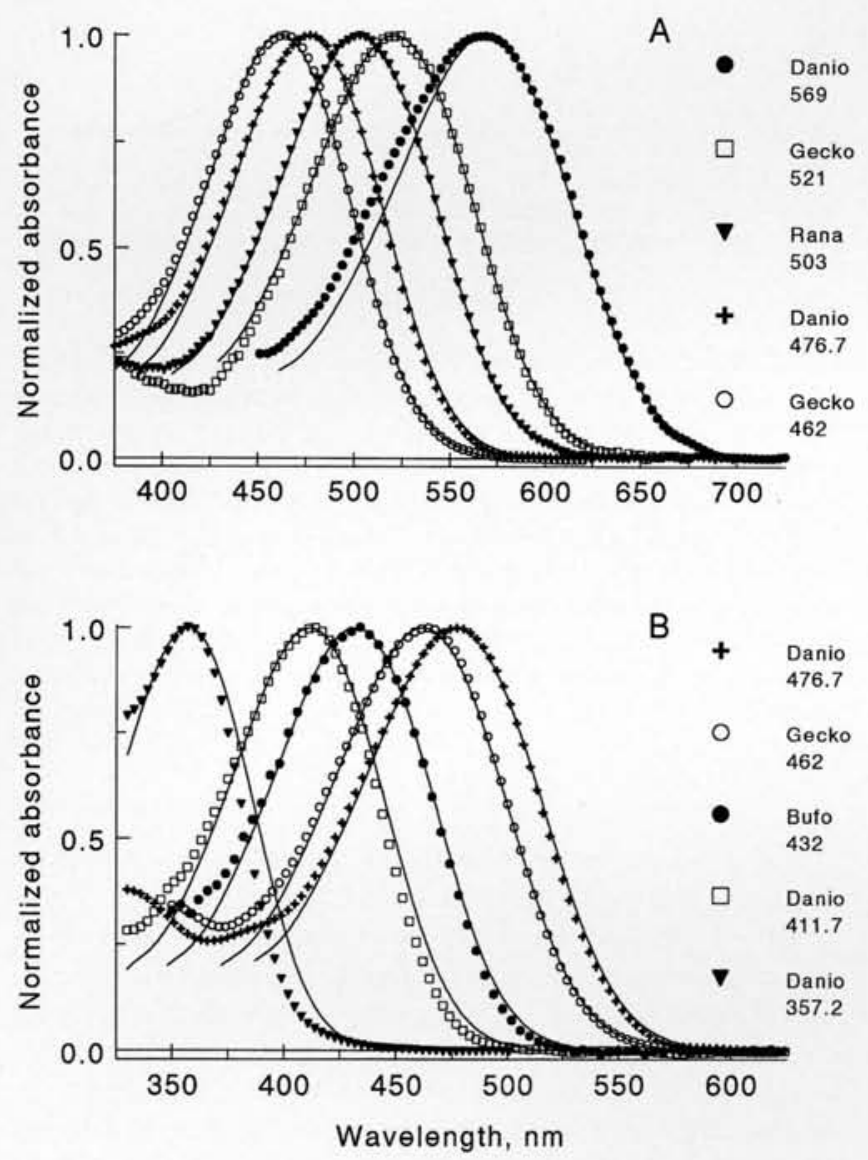

Fig. 3. MSP absorbance spectra of A1-based visual pigments (symbols) compared with the MM-transformed bovine template (smooth curves). All MSP spectra are averages of more than 25 outer segments, corrected for bleaching. The data for short-wave pigments have been Fourier filtered with 35 harmonics retained. For clarity, the eight different curves are displayed on two panels, so (A) comprises pigments with $\lambda_{\max }>462 \mathrm{~nm}$ and (B) pigments with $\lambda_{\max }<477 \mathrm{~nm}$; two curves are reproduced in both panels. Pigments with $\lambda_{\max }>500 \mathrm{~nm}$ show a good fit to the template, but the long-wave tails of more blue-sensitive pigments lie consistently below it (A), and the discrepancy increases with decreasing $\lambda_{\max }$ (B). small fish cones is inevitably of poorer quality than that from amphibian or gecko rods and contain obvious artifacts. For example, the elevated short-wave limb of the Danio red cone spectrum (Fig. 3A) can clearly be attributed to a strong local bleach during the scan and, hence, imperfect bleach correction (see Appendix 1). The effect of bleaching on the long-wave branch of absorbance spectra is negligible, however, and comparison should focus on this range. All the spectra with $\lambda_{\max }>500 \mathrm{~nm}$ in Fig. $3 \mathrm{~A}$ show rather a good agreement with the long-wave limb of the MMtransformed bovine template, whereas blue-sensitive Danio and gecko pigments consistently deviate from the template below halfmaximal absorbance. Fig. $3 \mathrm{~B}$ extends the comparison to even smaller $\lambda_{\max }$. All spectra in this panel (peaking below $500 \mathrm{~nm}$ ) show deviations, which are larger, the smaller is $\lambda_{\max }$. When the template is defined for best fit at peak absorbance, its long-wave limb runs markedly above the recorded spectrum. Similar deviations from the MM transform have been seen in MSP recordings of blue-sensitive pigments of deep-sea fish (Partridge \& De Grip, 1991; their Fig. 1) and the UV-sensitive pigment of zebrafish (Hárosi, 1994).

The systematic character of the deviations becomes evident when the slope of the middle part of the long-wave branch of each spectrum is plotted on a $\ln (\lambda)$ scale. The MM transform predicts that this slope be independent of $\lambda_{\max }$, but as shown in Fig. 4, in fact it increases when $\lambda_{\max }$ decreases from ca. 500 to $350 \mathrm{~nm}$. In the following section, we propose a modification of the MM transform to incorporate this trend.

\section{A template for the $\alpha$-band of Al pigments}

We start with an expression for the $\alpha$-band (i.e. the main absorbance band) of the form suggested by Lamb (1995):

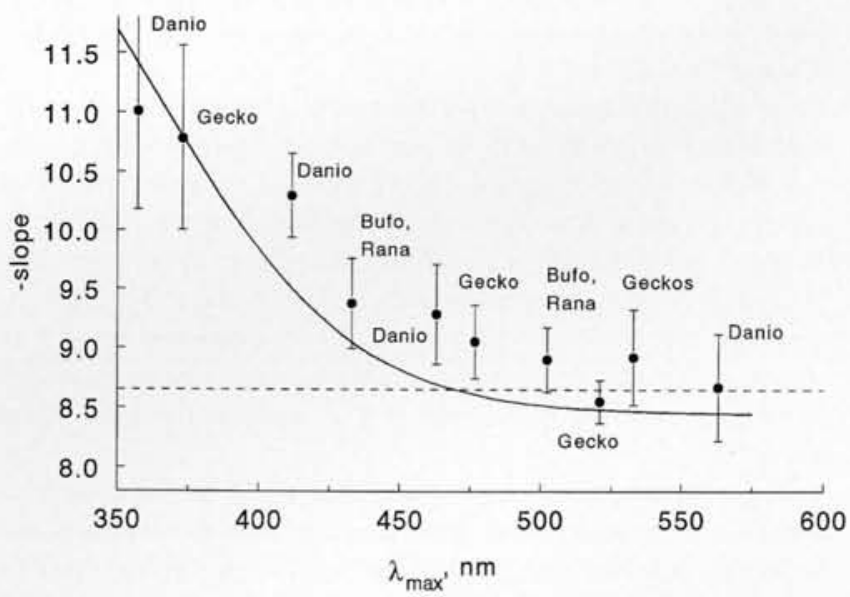

Fig. 4. Slope of the straight line fitting the middle part of the long-wave limb of the absorbance curve as plotted on a $\ln (\lambda)$ scale. The values for 10 types of $\mathrm{Al}$ pigments (including those considered in Fig. 3) are plotted as dots against the respective $\lambda_{\max }$ (average and 95\% confidence interval of several experimental sessions). Data on UV- and green-sensitive pigments in two extra gecko species added from Loew et al. (1996). The smooth curve shows the slope of the new template defined by eqns. (1) and (2) in the text. The horizontal dashed line shows the slope of the MM-transformed template based on bovine data. 
$S(x)=\frac{1}{\exp [A(a-x)]+\exp [B(b-x)]+\exp [C(c-x)]+D}$,

where $x=\lambda_{\max } / \lambda$. We found that a slight adjustment of Lamb's original parameter values gives an even better fit to the bovine data $(A=69.7, a=0.88, B=28, b=0.922, C=-14.9, c=1.104$, $D=0.674)$.

One advantage of Lamb's elegant formula is that it extends arbitrarily far into the long-wave end $(x \rightarrow 0)$, where it provides a good fit to physiologically recorded spectral sensitivities (which can be followed much farther into the red than absorbance). In this range, it reproduces the experimentally observed linear decrease of $\log S$ vs. $x$ (Griffin et al., 1947; Stiles, 1948). The parameters $A$ and $a$ determine the slope and $y$-intercept of this line. Under the MM transform, both these parameters are constant, ensuring the $\lambda_{\max }$ invariance of the long-wave slope. Since this invariance does not hold, at least one of them must be made a function of $\lambda_{\max }$. Em- pirically, we have found that a good fit is achieved by keeping $A$ constant and equal to 69.7 and setting

$$
a=0.8795+0.0459 \cdot \exp \left[-\left(\lambda_{\max }-300\right)^{2} / 11940\right] .
$$

For $\lambda_{\max }>500 \mathrm{~nm}$, eqn. (2) keeps $a$ virtually constant, but as $\lambda_{\max }$ decreases below that, $a$ starts growing progressively, increasing the long-wave slope. Eqn. (2) results in a good fit to the long-wave slope throughout entire $\lambda_{\max }$ range, for the expense of slightly worse fit to the middle part of the absorbance curve, between $70 \%$ and $30 \%$ maximum (cf. Fig. 4). However, the difference between the original and modified template in this region is almost imperceptible. Fig. 5 compares MSP spectra (same as in Fig. 3) with curves generated by eqns. (1) and (2), on linear (A) and logarithmic (B) ordinates. In all cases except one, the fit to the main part of the $\alpha$-band is quite good down to $0.01-0.001$ of maximum absorbance (where the data become too noisy for meaningful comparison, see panel B). The exception is the Danio UVsensitive cone, which deviates from the template by having a tail
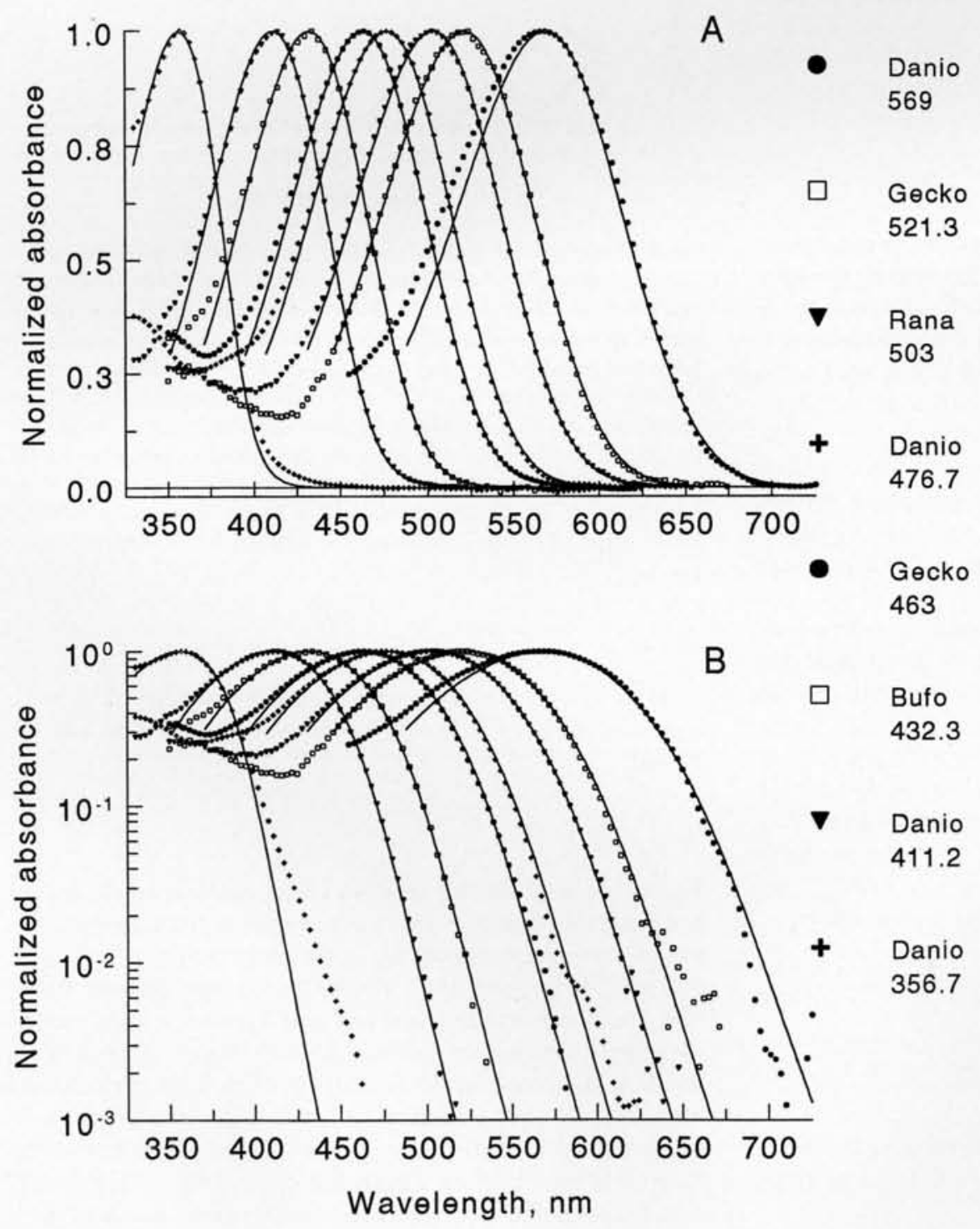

Fig. 5. Fit of the new template [eqns. (1) and (2) in the text; smooth curves] to the $\alpha$-band of the full range of A1-pigment absorbance spectra (symbols; same data as in Fig. 3). (A) linear, and (B) logarithmic ordinates. 
of high absorbance. This may indicate a mixture of two visual pigments (see Discussion).

Our modification also affects the determination of $\lambda_{\max }$ from the long-wave limb (MacNichol, 1986). Instead of the expression $\lambda_{\max }=\lambda_{0.5} / 1.0925$, which holds under the MM transform (see Methods), eqn. (2) requires

$$
\lambda_{\max }=45.02+0.756 \cdot \lambda_{0.5}+0.000141 \cdot \lambda_{0.5}^{2} .
$$

\section{The $\beta$-band of $A I$ pigments}

Another advantage of Lamb's formula is that its left limb "behaves well", providing a good fit for absorbances between 1.0 and 0.5 maximum and gradually falling to zero at short wavelengths. Thus, it outlines a distinct $\alpha$-band and provides a basis for decomposing the entire spectrum into $\alpha$ - and $\beta$-bands as suggested by Stavenga et al. (1993): the $\beta$-band is construed simply as the absorbance spectrum remaining after subtraction of the $\alpha$-band template from the complete experimental spectrum. The validity of this procedure critically depends on the quality of the data in the short-wave range. Our controls against some major error sources (light scattering, absorbance not due to visual pigment, the effect of bleaching products) are described in Appendix 2.

The short-wave peak remaining after subtraction of the $\alpha$-band template [eqns. (1) and (2)] was fitted with a Gaussian (Fig. 6):

$$
S_{\beta}(\lambda)=A_{\beta} \cdot \exp \left\{-\left[\left(\lambda-\lambda_{m \beta}\right) / b\right]^{2}\right\} .
$$

Here $A_{\beta}$ is the amplitude of the $\beta$-band relative to the $\alpha$-band, $\lambda_{m \beta}$ is the position of the $\beta$-peak, and $b$ is a bandwidth parameter. The parameter values were determined by a least-square fit. In green- and red-sensitive cells the $\beta$-peak was within the domain of recording $(\lambda>330 \mathrm{~nm})$ and all three parameters were left free for fitting (Figs. 6A and 6B). For amphibian green rods and Danio violet-sensitive cones, however, the $\beta$-band peaked below $330 \mathrm{~nm}$, and its amplitude could not be reliably determined. To avoid ambiguity, $A_{\beta}$ was fixed at the value 0.26 suggested by the best recordings from amphibian and gecko rods, and only $\lambda_{m \beta}$ and $b$ were left free (Fig. 6B, left curve). The $\beta$-band of UV-cones could not be characterized at all.

Fig. 7 summarizes the relationships between $\lambda_{\max }$ of the $\alpha$-band and the parameters of the $\beta$-band: $\lambda_{m \beta}, b$, and $A_{\beta}$. Because the data from different visual pigments were of uneven quality, the fits were carried out on data weighted by $\left[1 /\left(1-r^{2}\right)\right]$ where $r^{2}$ is the coefficient of determination of the Gaussian fit to the $\beta$-band. Further, the regression lines were constrained to pass through the most accurate point, which is the average for amphibian rhodopsins ( $\lambda_{\max }=502.6 \mathrm{~nm}, \lambda_{m \beta}=347.3 \mathrm{~nm}, b=57.5 \mathrm{~nm}$ ). As seen in the two top panels, the relationships between $\lambda_{\max }$ and $\lambda_{m \beta}$ and between $\lambda_{\max }$ and $b$ could be approximated as straight lines:

$$
\begin{aligned}
\lambda_{m \beta} & =189+0.315 \cdot \lambda_{\max }[\mathrm{nm}], \\
b & =-40.5+0.195 \cdot \lambda_{\max }[\mathrm{nm}] .
\end{aligned}
$$

The regression lines do not pass through the origin, which shows that the $\beta$-band does not obey the MM rule (cf. Stavenga et al., 1993).
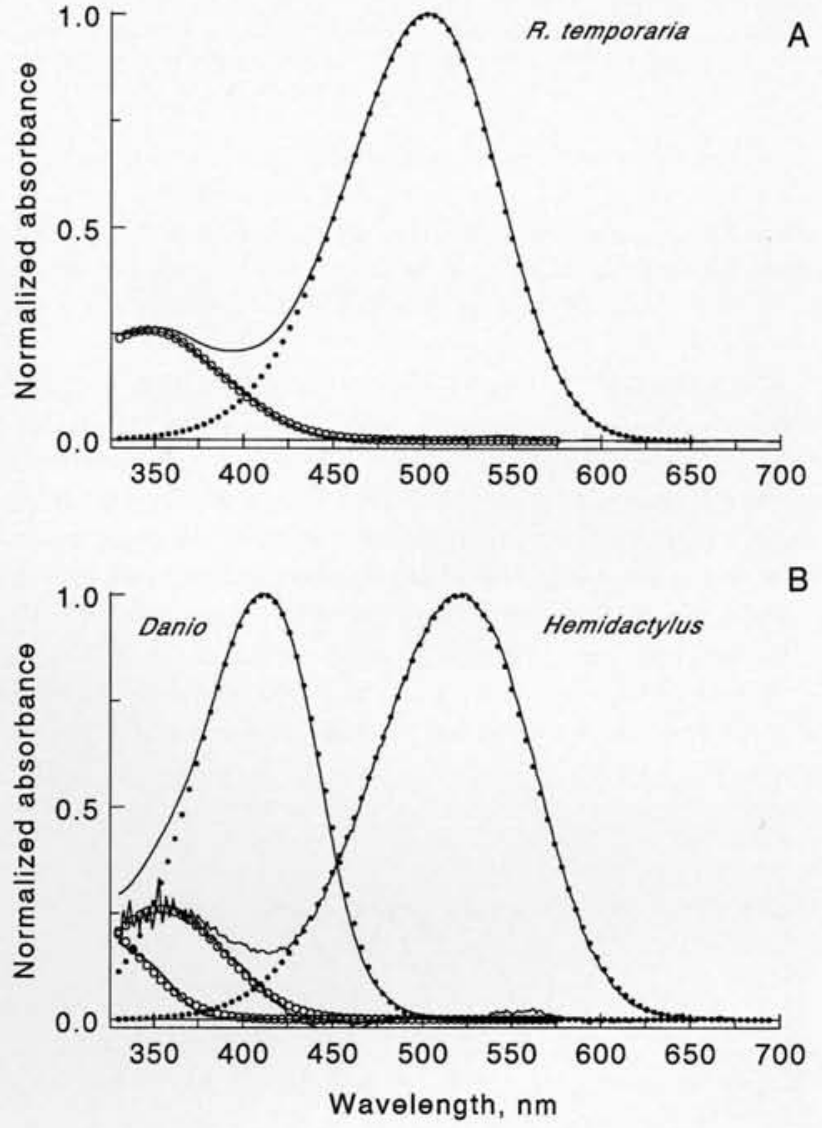

Fig. 6. Decomposition of the absorbance spectrum of $\mathrm{Al}$ pigments into $\alpha$ and $\beta$-bands. (A) MSP absorbance of rhodopsin rods in Rana temporaria (smooth curve), average of 29 OSs bleach corrected and Fourier filtered with 35 harmonics retained. Dots represent the best-fitting template ( $\lambda_{\max }=$ $502.6 \mathrm{~nm}$ ) generated according to eqns. (1) and (2). The open circles show the difference between the two curves. The smooth line through these is the best-fitting Gaussian [eqn. (4)], with amplitude $0.259, \lambda_{m \beta}=346 \mathrm{~nm}$ and $b=58 \mathrm{~nm}\left(r^{2}=0.9992\right)$. (B) The same decomposition procedure for the green-sensitive pigment of gecko and violet-sensitive pigment of Danio. Parameters: gecko, $A_{\beta}=0.26, \lambda_{m \beta}=354 \mathrm{~nm}, b=50 \mathrm{~nm}, r^{2}=0.992$; Danio, $A_{\beta}=0.26, \lambda_{m \beta}=307 \mathrm{~nm}, b=46.8 \mathrm{~nm}, r^{2}=0.98 . \lambda_{\max }$ as in Fig. 3.

Eqns. (1)-(5) provide a complete description of the absorbance spectra of A1-based visual pigments from about $330 \mathrm{~nm}$ into the far red.

\section{A2 pigments}

For A2 pigments, MM transforms of the template based on carp porphyropsin (Bridges, 1967) worked quite well for a broad sample of photoreceptors peaking in the range $440 \mathrm{~nm}<\lambda_{\max }<$ $540 \mathrm{~nm}$ : "green" and "red" rods of clawed toad Xenopus laevis, blue- and green-sensitive cones of carp Cyprinus carpio, rods of the sturgeons Acipenser güldenstädti and $A$. baeri $x A$. medirostris and blue-sensitive cones of A. stellatus (Fig. 8; the data on carp blue cones, Xenopus green rods and sturgeon rods have been omitted to avoid clutter). However, in more red-sensitive pigments the long-wave branch of the absorbance curve decays more steeply than predicted (Fig. 8, pigment-564 of A. stellatus and carp "red"- 

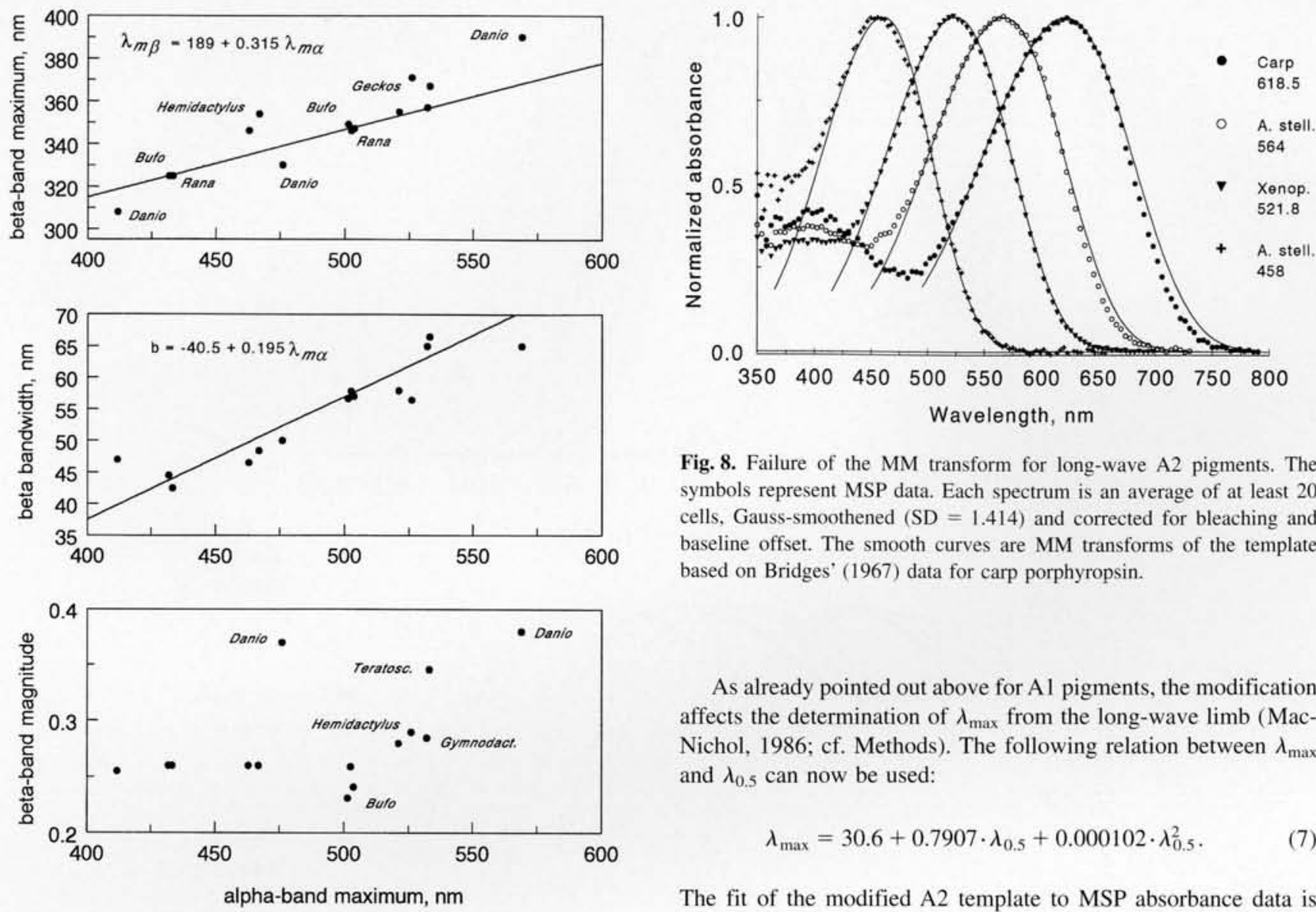

Fig. 7. Parameters of the $\beta$-band for all Al-based visual pigment studied, plotted against $\lambda_{\max }$ of the $\alpha$-band. Equations for the regression lines are given in the respective panels.

cone pigment). To incorporate these deviations from the MM transform into eqn. (1), it was necessary to make both the parameters $A$ and $a$ functions of $\lambda_{\max }$. For the purpose of finding the best expressions, we supplemented the long-wave tail of our absorbance data by published electrophysiological data on the spectral sensitivity of carp red-sensitive cones (Mooij \& van den Berg, 1983) and Ambystoma cones (Makino et al., 1990) (for correspondence between absorbance and sensitivity spectra, see Discussion). Fitting was carried out in two stages. First, a least-square fit was done to the Xenopus red rod spectrum from $450 \mathrm{~nm}$ upwards, with all seven parameters in eqn. (1) free for fitting but $\lambda_{\max }$ fixed at $523 \mathrm{~nm}$. Then all the other pigments were fitted with only $A, a$, and $\lambda_{\max }$ as free parameters. The functions $A\left(\lambda_{\max }\right)$ and $a\left(\lambda_{\max }\right)$ obtained were approximated by exponential functions, the parameters of which were determined by the method of least squares. The set of parameters in eqn. (1) that provide a good fit to the full range of our $\mathrm{A} 2$ data is $B=20.85, b=0.9101, C=-10.37, c=$ $1.1123, D=0.5343$, and

$$
\begin{aligned}
& A=62.7+1.834 \cdot \exp \left[\left(\lambda_{\max }-625\right) / 54.2\right], \\
& a=0.875+0.0268 \cdot \exp \left[\left(\lambda_{\max }-665\right) / 40.7\right] .
\end{aligned}
$$

Fig. 8. Failure of the MM transform for long-wave A2 pigments. The symbols represent MSP data. Each spectrum is an average of at least 20 cells, Gauss-smoothened $(\mathrm{SD}=1.414)$ and corrected for bleaching and baseline offset. The smooth curves are MM transforms of the template based on Bridges' (1967) data for carp porphyropsin.

As already pointed out above for $\mathrm{Al}$ pigments, the modification affects the determination of $\lambda_{\max }$ from the long-wave limb (MacNichol, 1986; cf. Methods). The following relation between $\lambda_{\max }$ and $\lambda_{0.5}$ can now be used:

$$
\lambda_{\max }=30.6+0.7907 \cdot \lambda_{0.5}+0.000102 \cdot \lambda_{0.5}^{2} .
$$

The fit of the modified A2 template to MSP absorbance data is shown in Fig. 9 on linear (A) and logarithmic (B) ordinates.

In the same way as for A1 pigments, the full absorbance spectrum was decomposed into $\alpha$ - and $\beta$-bands (Fig. 10). For $\lambda_{\max }>$ $520 \mathrm{~nm}$, the $\beta$-peak was within the measured range and we fitted the $\beta$-band with eqn. (5) leaving all three parameters $\left(A_{\beta}, \lambda_{m \beta}\right.$, and $b$ ) free. For short-wave pigments where the $\beta$-peak was outside the measured range, $A_{\beta}$ was fixed at 0.37 . The parameter values obtained are plotted against $\lambda_{\max }$ in Fig. 11. The relationship between $\lambda_{m \beta}$ and $\lambda_{\max }$ is again approximately linear (top panel), while $b$ versus $\lambda_{\max }$ now required a second-order approximation (middle panel):

$$
\begin{aligned}
\lambda_{m \beta} & =216.7+0.287 \cdot \lambda_{\max }[\mathrm{nm}] \\
b & =317-1.149 \cdot \lambda_{\max }+0.00124 \cdot \lambda_{\max }^{2}[\mathrm{~nm}] .
\end{aligned}
$$

The amplitude of the $\beta$-band shows no correlation with $\lambda_{\max }$ (bottom panel).

The absorbance maxima of all visual pigments studied, both A1- and A2-based, and the types of cells housing them are summarized in Table 1.

\section{Discussion}

\section{Reliability of MSP data}

MSP is used for measuring the absorbance spectrum of a visual pigment in situ, usually in order to obtain the physiological spectral sensitivity of the photoreceptor cell. This raises important questions about reliability and functional relevance. Does the re- 


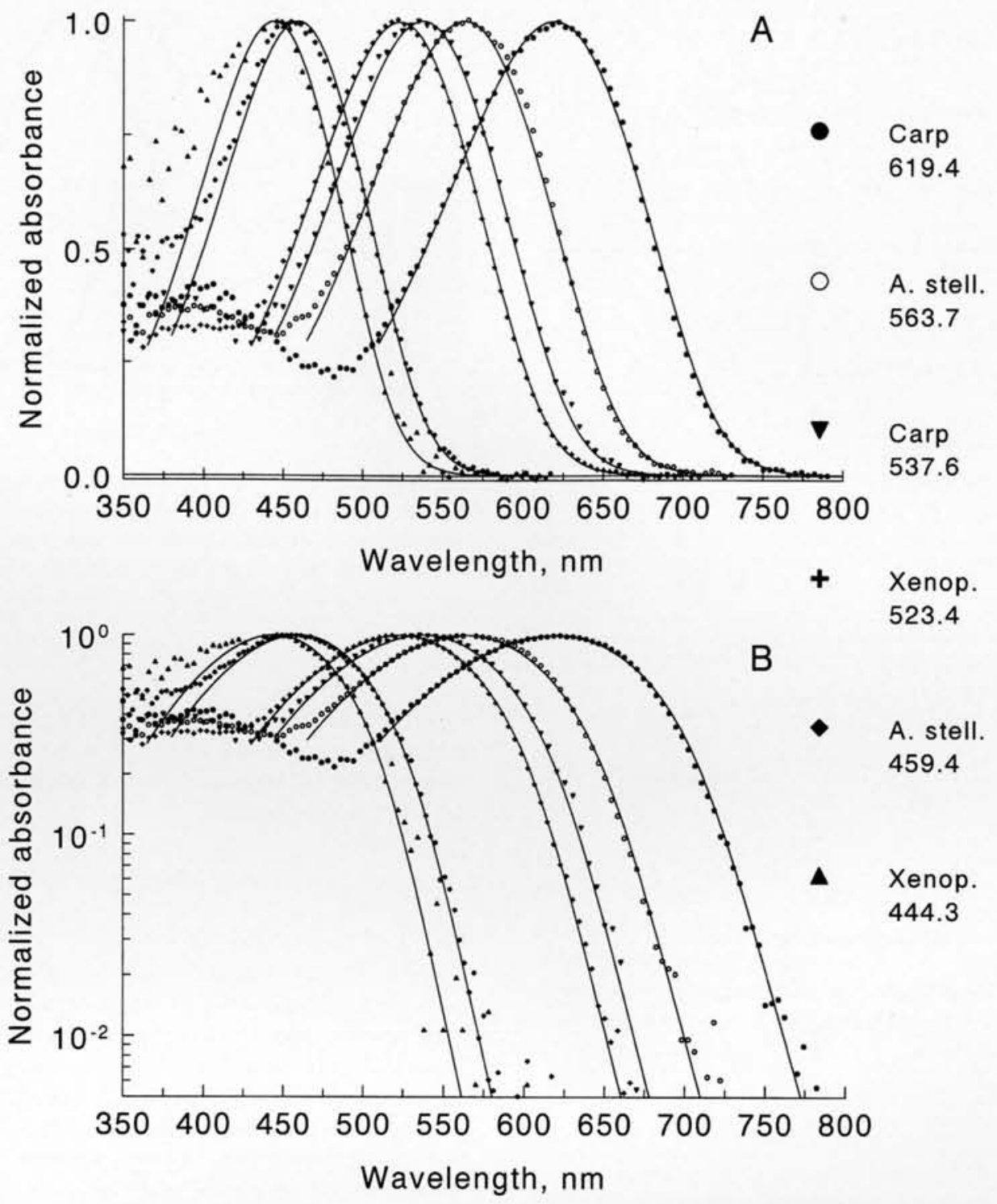

Fig. 9. Fit of the new A2-template (smooth curves) to the $\alpha$-band of the full range of A2-pigment absorbance spectra (symbols). The template was generated according to eqns. (1) and (6) in the text. (A) linear, and (B) logarithmic ordinates.

corded spectrum truly represent the light-absorbing properties of the OS? Is the absorption entirely due to the visual pigment, or are other light-absorbing substances present in sufficient amounts to significantly compromise the data? We shall first consider these more or less technical problems, leaving to the following section the question whether spectral sensitivity can be assumed to exactly follow the proportion of light absorbed by the visual pigment.

Sources of error in MSP have been discussed in detail by Liebman (1972), Hárosi (1975a), Cornwall et al. (1984), Levine and MacNichol (1985), and MacNichol (1986). In our experiments on the big rods of amphibians many of the error sources are reasonably well controlled (Appendices 1 and 2). Our correction for bleaching during the scan, the fact that $\alpha$-band absorbance could be virtually completely abolished by bleaching, and the straight and nearly horizontal zero line imply that pigment bleaching, lightinsensitive substances and optical artifacts, respectively, can have affected the spectra only marginally at wavelengths $>440 \mathrm{~nm}$ (see
Fig. 16, and Fig. 17 in the Appendix 1). Further, recordings with two orientations of the polarizer show that the dichroism of the photoreceptor membrane is constant down to $375 \mathrm{~nm}$ (Fig. 17B). This fits the idea that the absorption between 440 and $375 \mathrm{~nm}$ is also completely due to rhodopsin, and that the structures underlying $\alpha$ - and $\beta$-band absorbance are similarly oriented with respect to the membrane plane. At shorter wavelengths, however, there was a clear indication of variable UV absorbance not due to rhodopsin (see Fig. 17B), but controls for this were done only in amphibian rhodopsin rods and green-sensitive receptors of geckos. Elevated absorbance in the blue/UV end is evident also in most previously published spectra, whether from MSP or pigment extracts. It could result from varying amounts of an "end-absorbing" pigment supposedly present in OSs (Dartnall, 1961; Cornwall et al., 1984), if not from noncorrected optical problems and bleaching. Thus, MSP data in the $\beta$-band region should be viewed with some caution. 


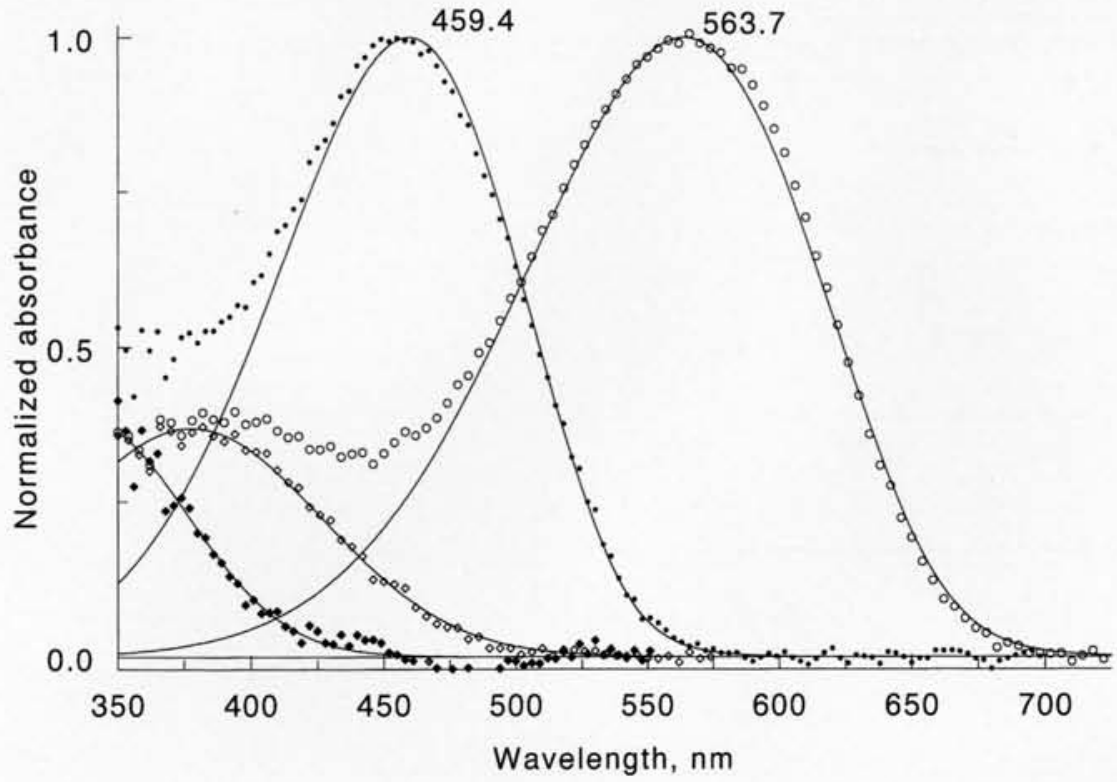

Fig. 10. Decomposition of the absorbance spectrum of A2 pigments into $\alpha$ - and $\beta$-bands. MSP data from cones of A. stellatus: red-sensitive (open circles), blue-sensitive (dots). Each spectrum is an average from 20 cells, bleach corrected and Gauss filtered. The smooth curves are the best-fitting templates for the $\alpha$-band $\left(\lambda_{\max }=563.7 \mathrm{~nm}\right.$ and $\left.459.4 \mathrm{~nm}\right)$ generated according to eqns. (1) and (6). Diamonds plot the difference between MSP and template; the smooth lines through the diamonds are the best-fitting Gaussians [eqn. (4) in the text]. Parameters: red-sensitive cones, $A_{\beta}=0.37, \lambda_{m \beta}=378 \mathrm{~nm}, b=69 \mathrm{~nm}\left(r^{2}=\right.$ 0.972 ); blue cones, $A_{\beta}=0.38, \lambda_{m \beta}=339 \mathrm{~nm}, b=$ $52.4 \mathrm{~nm}\left(r^{2}=0.97\right)$.

An extra complication can be expected with small cone OSs. The use of a low-NA condenser (see Methods) to reduce light scatter degrades spatial resolution instead. The central image of the measuring beam is surrounded by secondary diffraction maxima, and with decreasing NA these are spaced more widely and draw more light from the main spot. Moreover, the distance between the central and secondary maxima is wavelength dependent. If the diffraction pattern is too wide, the fraction of light that passes through the OS and its highly refracting border will vary with wavelength, producing spurious spectral "absorbance". We have a good control against this only in UV- and violet-sensitive cones, where the long-wave tail of the spectrum was straight and almost horizontal from $450 \mathrm{~nm}$ (UV cone) or $500 \mathrm{~nm}$ (violet cone) up to
$750 \mathrm{~nm}$. The situation is much less certain for the blue- and redsensitive Danio cones with their smaller OSs and shorter available stretch of zero line. Thus, a certain distortion of the spectra recorded from these cells cannot be excluded at present.

\section{Absorbance and spectral sensitivity}

Our MSP spectra of Al-based visual pigments with $\lambda_{\max }>480 \mathrm{~nm}$ were well fit by a template based on bovine extract data plus the $\mathrm{MM}$ transform. This is at variance with recent electrophysiological results by Palacios et al. (1998a) from rhodopsin rods of Rana pipiens and Ambystoma tigrinum, which showed significantly narrower spectra than mammalian rhodopsin templates. Fig. 12 illus-

Table 1. Absorbance maxima and chromophoric group of the visual pigments studied ${ }^{a}$

\begin{tabular}{|c|c|c|c|c|c|c|c|c|}
\hline \multirow[b]{2}{*}{ Species } & \multirow[b]{2}{*}{ Chromophore } & \multicolumn{2}{|c|}{ Rods } & \multicolumn{5}{|c|}{ Cones } \\
\hline & & "red" & "green" & UV & V & B & G & $\mathrm{R}$ \\
\hline Danio aequipinnatus & Al & & & 357 & 411 & 477 & & 569 \\
\hline Brachydanio rerio & Al & & & 361 & 414 & 483 & & 567 \\
\hline Rana temporaria & Al & 503.4 & 434 & & & NR & & \\
\hline R. ridibunda & Al & 502.1 & 433 & & & NR & & \\
\hline R. catesbeiana & Al & 501.6 & 432 & & & NR & & \\
\hline R. pipiens & Al & 502.5 & NR & & & NR & & \\
\hline Bufo bufo & Al & 501.7 & 432 & & & NR & & \\
\hline B. marinus & Al & 503.3 & 432 & & & NR & & \\
\hline Hemidactylus frenatus & $\mathrm{Al}$ & & & NR & & 463 & 521 & \\
\hline Gymnodactylus caspius & Al & & & NR & & 467 & 532 & \\
\hline Acipenser stellatus & $\mathrm{A} 2$ & & & & & 459 & & 564 \\
\hline A. güldenstädti & A2 & & & & & NR & 537 & NR \\
\hline A. baeri $x$ A. medirostris & $\mathrm{A} 2$ & & & & & NR & NR & NR \\
\hline Cyprinus carpio & A2 & & & NR & & 452 & 538 & 619 \\
\hline Carassius auratus & $\mathrm{A} 2$ & & & NR & & 451 & 539 & $602-614$ \\
\hline Xenopus laevis & $\mathrm{A} 2$ & 523 & 444 & & & NR & & \\
\hline
\end{tabular}

${ }^{\mathrm{a}} \mathrm{NR}=$ not recorded during present study but known to be present in the species. Dash: lacking cell type. Cone types: UV, ultraviolet; V, violet; B, blue; $\mathrm{G}$, green; $\mathrm{R}$, red absorbing. 

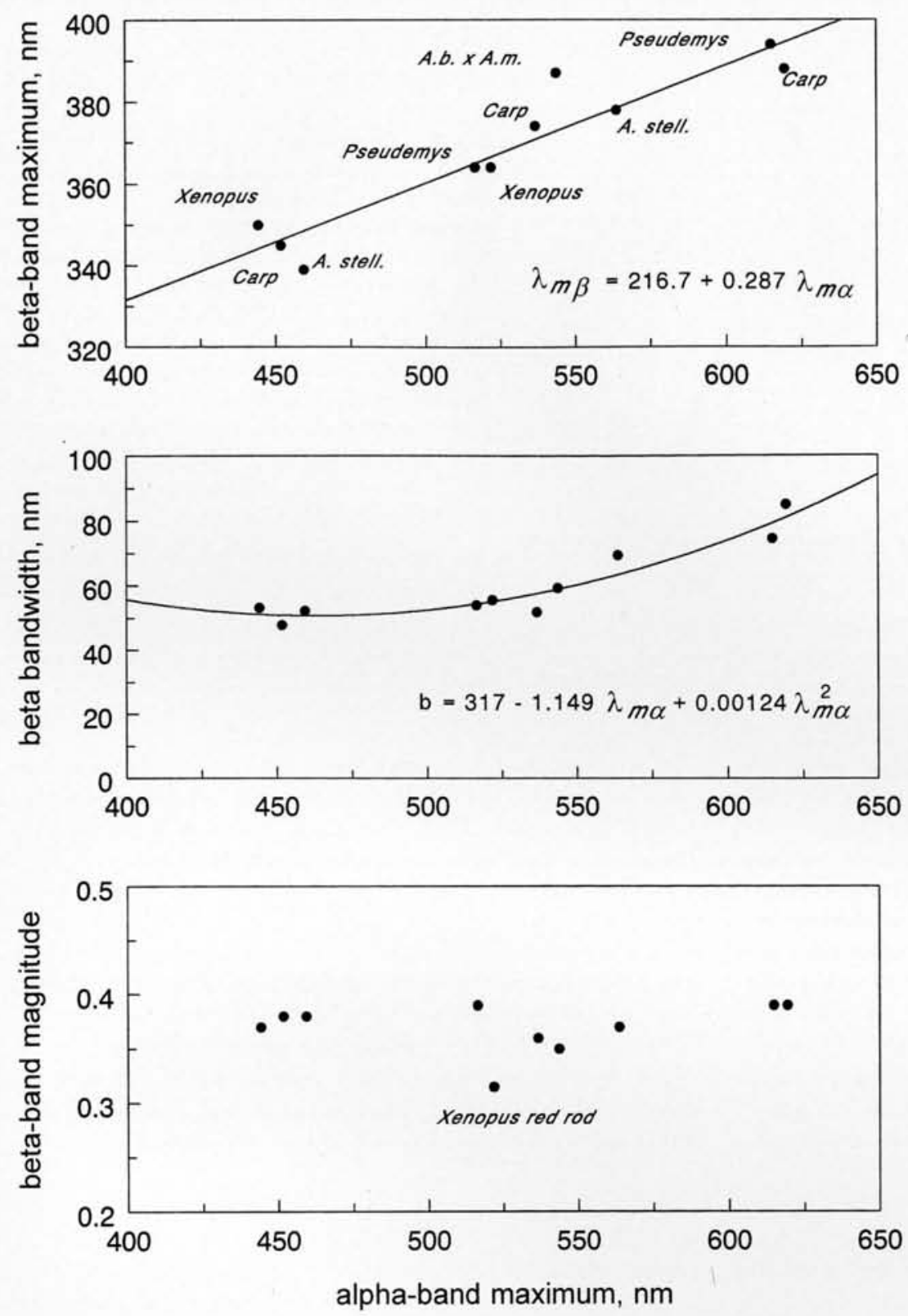

Fig. 11. Parameters of the $\beta$-band for all A2-based visual pigment studied, plotted against the position of the absorbance maximum ( $\lambda_{\max }$ ) of the $\alpha$-band. Data for the turtle, Pseudemys scripta elegans, from Loew and Govardovskii (unpublished). Equations for the fits to the data are given in corresponding panels. trates the same discrepancy between their $R$. pipiens sensitivity data (open circles) and our present amphibian absorbance data (dots, $R$. temporaria). The species difference is not a likely explanation, because all five anuran species studied here had virtually identical curve shapes, with only a slight $(<3 \mathrm{~nm})$ difference in $\lambda_{\max }$. Moreover, rhodopsin extracts from adult $R$. pipiens (Donner \& Reuter, 1976, and unpublished) yield spectra that agree closely with those of other frogs and with the bovine-based template.

A possible explanation would be that spectral sensitivity does not correlate exactly with absorbance. Though the "principle of univariance" (Naka \& Rushton, 1966) is firmly founded in both experiments and photochemical laws, it is not absolutely impossible that the quantum yield of photoreceptor excitation could vary slightly even within the main absorption band. Among the scarce spectral-sensitivity data [other than those of Palacios et al. (1998a)] suitable for comparisons with absorbance in amphibian rods, the suction-pipette recordings from larval Ambystoma tigri- num by Cornwall et al. (1984) are particularly relevant. Unlike a large body of measurements on cells embedded in retinal tissue (eyecup or isolated retina), they are comparatively impervious to optical problems such as light scattering, prereceptor filtering, waveguide effects, and rhodopsin self-screening. The authors measured both absorbance and spectral sensitivity in the same set of porphyropsin-containing rods of Ambystoma, with the same instrument adapted for both MSP and suction-pipette recording. They found excellent agreement for $\lambda>440 \mathrm{~nm}$, although below this, absorbance rose markedly above sensitivity (and above our A2 template), which could be attributed to optical problems and/or extra absorbing materials (Cornwall et al., 1984). We further compared their spectral-sensitivity curve with our absorbance data on Xenopus rods containing virtually identical visual pigment, and found good agreement over the entire $\alpha$-band (400$700 \mathrm{~nm}$; not shown). Thus these comparisons provide no support for the idea of variable quantum yield, and the cause of the 

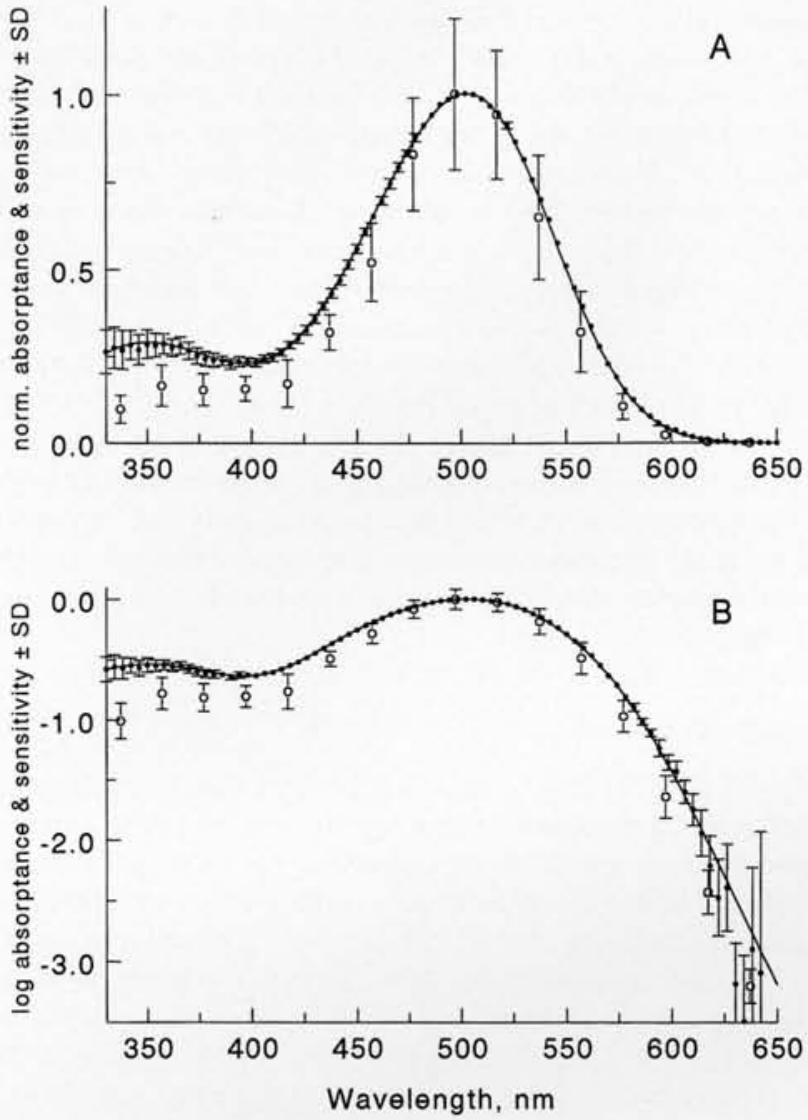

Fig. 12. Comparison of the spectral sensitivity data from Rana pipiens rhodopsin rods by Palacios et al. (1998a) (open circles) with our MSP absorbance data from $R$. temporaria (dots) on linear (A) and logarithmic (B) ordinates. The MSP data are bleach-corrected averages from 18 OSs. The raw spectra were weakly filtered by convolution with a Gaussian $(\mathrm{SD}=1.414 \mathrm{~nm})$ and normalized at peak prior to averaging. The continuous line is the template with $\lambda_{\max }=502.6 \mathrm{~nm}$. MSP absorbance values have been converted into absorptances $\left(\mathrm{OD}_{\max }=0.1\right)$ to allow direct comparison with spectral sensitivity.

narrow sensitivity spectra obtained by Palacios et al. (1998a) remains unknown.

\section{MSP and electrophysiology}

Accepting that the spectral sensitivity of the photoreceptor cell exactly parallels the proportion of light absorbed by the visual pigment, MSP and electrophysiology represent two alternative methods for determining absorbance/sensitivity spectra. Each has its own range of excellence. Inspection of the error bars in Fig. 12 shows that, at least in favorable cases like the big rods of amphibians, MSP outperforms single-cell electrophysiology over the main part of the curve. MSP produces an essentially continuous set of data, and a hundred spectra can be recorded during a single experimental day, which allows substantial improvement by averaging. Collecting similar statistics just for a few points on a physiological spectral-sensitivity function could take weeks. Thus, MSP is the method of choice for assessing spectra down to some $0.1-1 \%$ of maximum. In many applications, this may be quite enough. For instance, in most natural illuminations, the contribution of very long wavelengths to the photoreceptor re- sponse is negligible, so many computations related to visual ecology and color vision in natural conditions may completely rely upon spectra characterized by MSP.

At longer wavelengths, where absorbance is very low, MSP fails completely and physiological measurement of spectral sensitivity is the only possibility. Templates designed to be valid over the whole spectral range must be based on both types of data properly "glued" together. This, of course, does not imply that the template itself must necessarily consist of "glued" components, as for polynomial or log-normal curves (cf. Mooij \& van den Berg, 1983; Stavenga et al., 1993; Palacios et al., 1996, 1998a,b).

\section{Template design and modification of the MM transform}

The very idea of a template demands that it should be valid for visual pigments from different sources, covering the entire range of $\lambda_{\max }$. Otherwise, fitting a particular set of experimental data with a specific mathematical expression only provides a means to interpolate within the set and has no predicting power outside it, either in the wavelength or in the "species" domain. Thus, a standard type of expression with a fixed set of parameters should be chosen, and its applicability tested for as wide a range of visual pigments as possible.

The specific analytical form chosen in the first place is a matter of convenience, and a number of different forms have been successfully used: a sum of Gaussians (Hárosi, 1976), polynomials (Dawis, 1981; Baylor et al., 1984, 1987; Makino et al., 1990; Kraft et al., 1993; Makino \& Dodd, 1996; Palacios et al., 1996, 1998a,b), a combination of trigonometric and exponential functions (Maximov, 1988), Chebyshev polynomials (Partridge \& De Grip, 1991), a log-normal function (Lewis, 1955; Metzler \& Harris, 1978; Mooij \& van den Berg, 1983), and a modified log-normal function (Stavenga et al., 1993). However, all polynomial templates, as well as the log-normal function, have the flaw that they are well behaved only within a limited wavelength range.

We have basically adopted Lamb's (1995) formulation [eqn. (1)] because of several attractive features: it provides an accurate description of the main part of the $\alpha$-band as measured by MSP, it decays exponentially at very long wavelengths in accordance with physiological data, and it goes to zero on the short-wave side, thus naturally outlining an $\alpha$-band. With constant parameters, it implements the MM transform and (with one set of parameter values) provides an excellent description of data in the wavelength range $\lambda>0.9 \lambda_{\max }$ for A1 pigments with $\lambda_{\max }>480 \mathrm{~nm}$ (Lamb, 1995; cf. our Fig. 3A). With a different set of constant parameter values, it provides a good fit to A2 pigments peaking at intermediate and short wavelengths (Fig. 8).

In response to the systematic deviations from the MM transform observed for short-wavelength-sensitive A1 pigments $\left(\lambda_{\max }<\right.$ $480 \mathrm{~nm}$, cf. Fig. 3B) and long-wavelength-sensitive A2 pigments ( $\lambda_{\max }>550 \mathrm{~nm}$, cf. Fig. 8), we have chosen to retain the general form of the Lamb template, but to make one or two of the parameters vary with $\lambda_{\max }$ [ $A$ and a in eqn. (1), as expressed by eqns. (2) and (6)]. This preserves the main properties of the template, but makes it reproduce the spectral narrowing observed in the pigments at "extreme" positions (Figs. 5 and 9).

\section{Quality of fit of the modified templates at long wavelengths}

\section{Al template}

In Figs. 5 and 9, our modified templates were confronted with experimental data only over the ranges where absorbance was high 
enough to be measured by MSP. Their applicability, however, also depends on performance on the long-wavelength slope which can only be studied by electrophysiology. In Fig. 13, our modified A1 template [eqns. (1) and (2)] is thus compared with the rather small number of suitable sensitivity spectra that are available from single photoreceptors peaking at wavelengths where the MM transform fails: UV-, violet-, and blue-sensitive cones of Danio, macaque, and Ambystoma (see figure legend for references). The fits to the long-wave tails are seen to be fair to excellent, indicating that our modification of the MM transform, originally based on MSP data, does hold far beyond the range accessible by MSP. For these data, however, the apparent agreement at the top of the curves $(0.05-1.0$ maximum) is somewhat deceptive, since the logarithmic ordinate compresses this part of the graph. On a linear plot, irregular deviations of electrophysiological sensitivities from the template as well as from MSP data would be evident.

\section{A2 template}

For A2 photoreceptors, there is even less spectral-sensitivity data of suitable quality. The goldfish results of Palacios et al. $(1998 b)$ cannot be used, because individual fish have varying A2/A1 mixtures. Still, our MSP spectra from green- and blue-sensitive cones fit theirs rather well. However, our MSP spectra from redsensitive cones varied greatly and, above all, were mostly significantly different from those reported by Marks (1965), Hárosi and MacNichol (1974a), Hárosi (1976), and Tsin et al. (1981) (data not shown). This may indicate genetic polymorphism (Johnson et al., 1993) and possibly co-expression of several opsins within the same cone, making the goldfish an inappropriate animal for establishing standards.

The only suitable data from isolated A2 photoreceptors known to us are those of Cornwall et al. (1984) on Ambystoma rods,
Palacios et al. (1998a) on Xenopus rods, and Makino et al. (1990) on Ambystoma cones, shown in Fig. 14. The figure also includes carp sensitivity functions based on the ERG recordings of Mooij and van den Berg (1983), where, admittedly, the exact relation to pigment absorbance is less certain than for suction-pipette recordings. It can be seen that the Palacios et al. (1998a) spectrum from Xenopus rods (open diamonds with error bars) is again somewhat narrower than our template and MSP spectrum, although the deviations are smaller than for their data on $R$. pipiens rhodopsin rods (cf. Fig. 12). When optimizing the parameters of our template for long-wavelength-sensitive A2 pigments [eqns. (6a, 6b)], we actually supplemented our MSP with spectral-sensitivity data from red-sensitive cones of Ambystoma and carp. The template fit to the long-wavelength slope is accordingly good, as is true, of course, also for the photoreceptors where our modification of the MM transform makes little difference (carp rods and blue-sensitive cones of Ambystoma).

\section{Transformation rule}

Fig. 15 provides final comparison between different transforms used until now to describe visual pigment spectra with $\lambda_{\max }$ distant from $500 \mathrm{~nm}$ (for A1-based pigments). Since our template describes available experimental data on absorbance and sensitivity quite accurately (cf. Figs. 1, 2, 5, 6, and 13), we use it as a basis for comparison (dots in Fig. 15). Dartnall's original transform (shape invariance in the frequency scale) generates curves that are too wide for the long-wave visual pigments, and too narrow for the short-wave ones. Ebrey and Honig (1977) nomogram [and Dawis' (1981) formula that implemented the same idea] was the first attempt to overcome the difficulty. Unfortunately, their solution was unsatisfactory because it used piecewise approximation of an

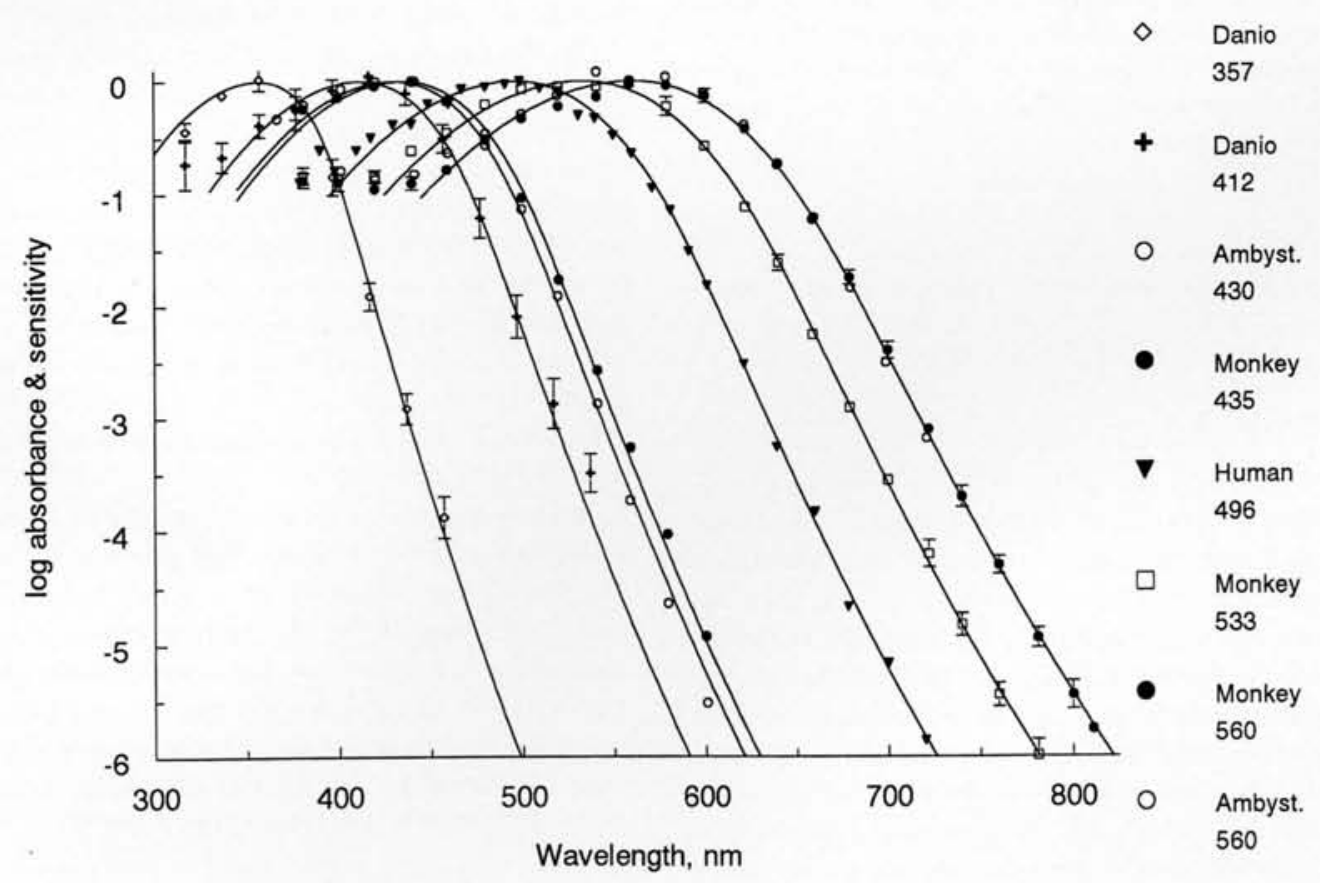

Fig. 13. Comparison of the new A1-template (continuous curves) with spectral-sensitivity data obtained by suction pipette recordings from isolated human rods (Kraft et al., 1993), Danio UV- and violet-sensitive cones (Palacios et al., 1996), macaque cones (Baylor et al., 1987), and Ambystoma cones in which the native A2 chromophore had been substituted by 11-cis retinal (Makino et al., 1990; tabulated data kindly supplied by Clint Makino). The symbols marking the spectral-sensitivity data are identified in the figure. SD bars are shown when available in published tables. 


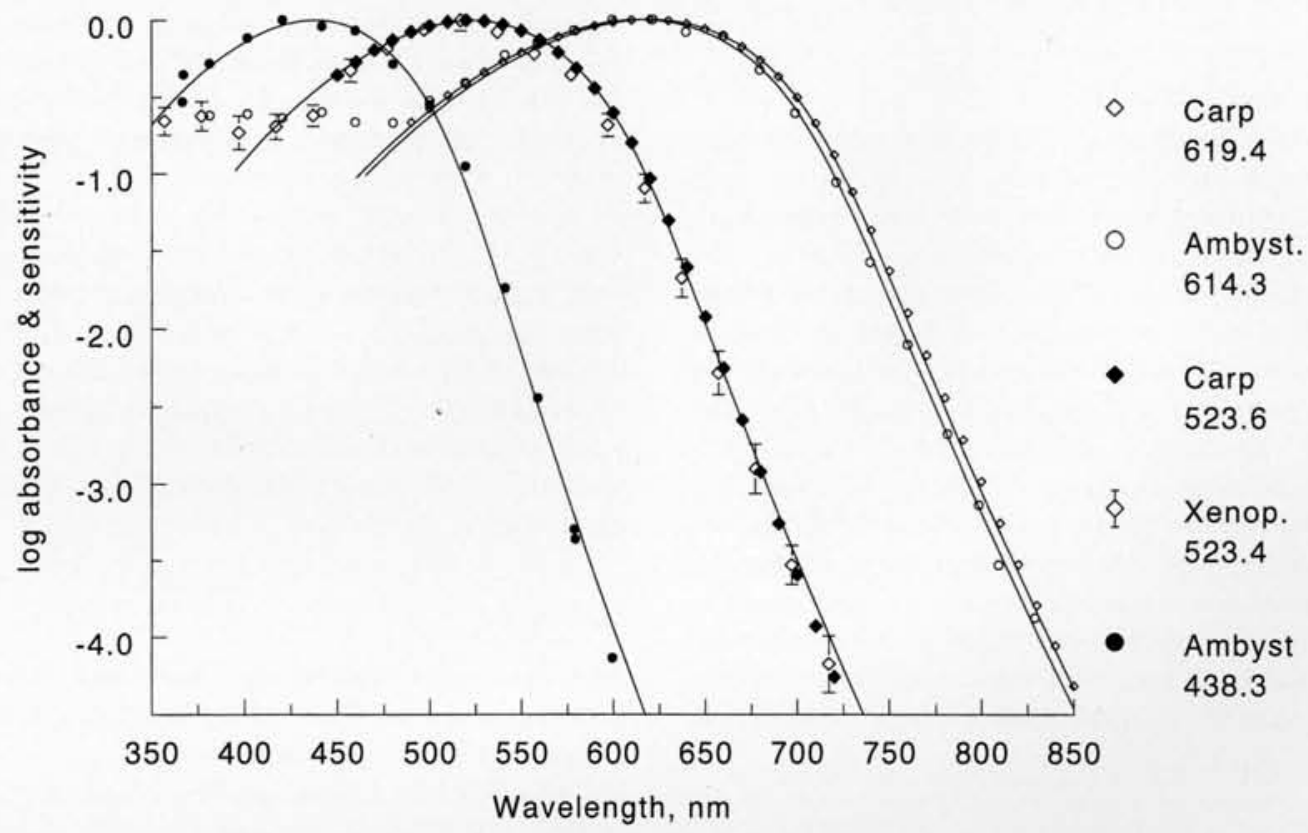

Fig. 14. Comparison of the new A2-template (continuous curves) with spectral-sensitivity data obtained by suction pipette recordings from isolated Xenopus rods (Palacios et al., 1998a) and Ambystoma cones with native A2-chromophore (Makino et al., 1990), and by ERG recording from carp rods and red-sensitive cones (Mooij \& van den Berg, 1983). The data symbols are identified in the figure. [Since the carp data are not tabulated in the Mooij \& van den Berg (1983) paper, the sensitivities shown have been derived from the equations used by the authors to describe their results (see their Figs. 3A and 3D)].

essentially continuous transformation function, and yielded ambiguous values at the borders between pieces. Thus we do not include it in the comparison. The $\lambda^{1 / 4}$ transform originally suggested by Barlow (1982) works better but also fails both at short and long wavelengths. MM transform (shape invariance in the normalized wavelength/ frequency scale) is apparently perfect for $\lambda_{\max }>$ $465 \mathrm{~nm}$, but progressively fails for more short-wave pigments (cf. also Figs. 3 and 5).

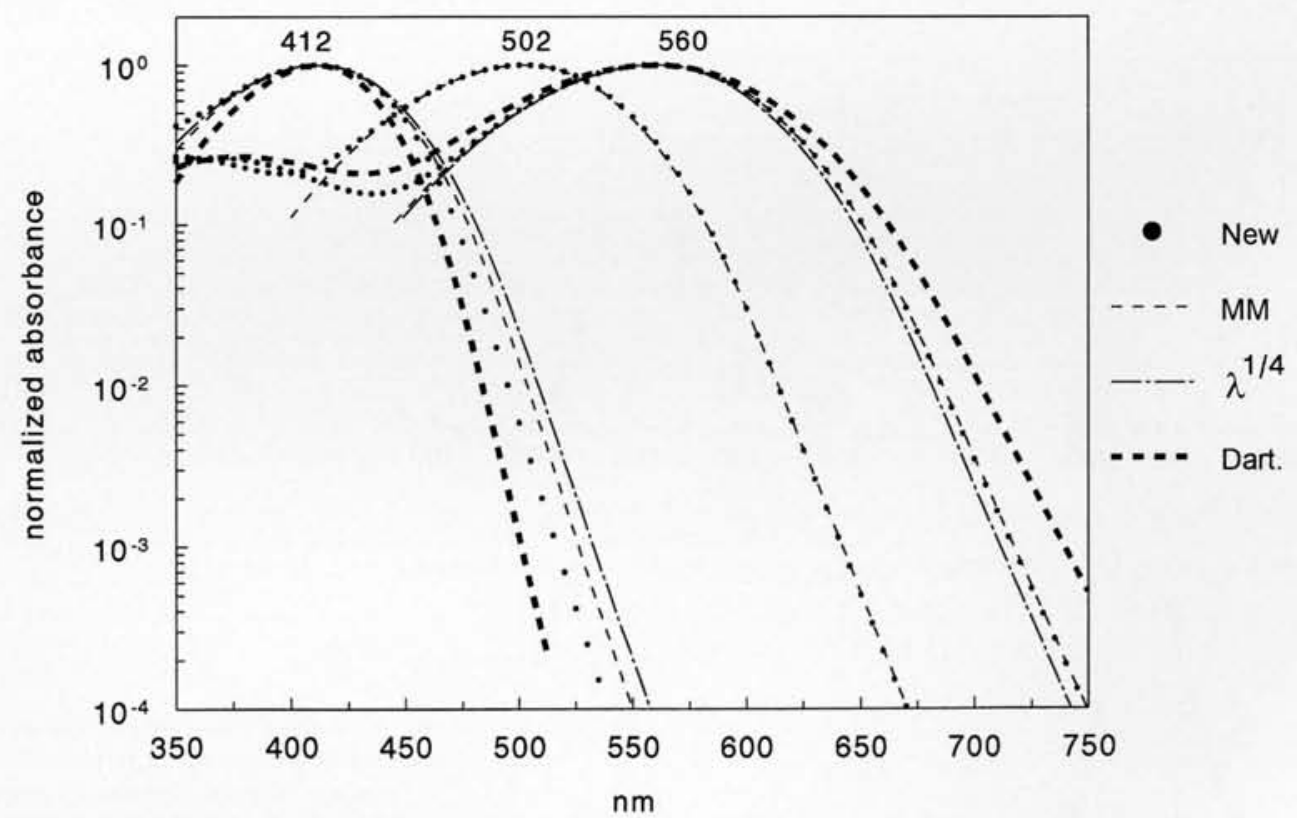

Fig. 15. Comparison of different transforms applied to the same basic rhodopsin curve. The absorbance curve of rhodopsin-500 (dots) was generated by the eqn. (1) in the text and then transformed to $\lambda_{\max }=400$ and $575 \mathrm{~nm}$. Thick hyphenated line, Dartnall's rule (shape invariance in the frequency scale); thin hyphenated line, MM transform (normalized frequency/wavelength scale); dotted hyphenated line, $\lambda^{1 / 4}$ scale; dots, present paper, eqns. (1)-(2). 


\section{The $\beta$-band}

\section{Accuracy of $\beta$-band estimates}

Results on the $\beta$-band are not as accurate as those for the main $(\alpha-)$ absorption band. Recording noise is high due to low lamp output in the UV, but more importantly, there are substantial differences between published absorbance data for $\lambda<400 \mathrm{~nm}$. The error sources already discussed are most obnoxious in this spectral range, and in addition the accumulation of bleaching products (mainly metarhodopsin II) is difficult to control and depends, for example, on beam size and the speed of the spectral scan. Our bleach correction (Appendix 1) and other controls (Appendix 2, Fig. 18) largely rule out these errors in the data from toad and frog rods, as is suggested also by the modest amplitude variation of the $\beta$-peak in these cells (range: $24-26 \%$ of the $\alpha$-peak: see Fig. 7). For long-wave cones, however, some controls are lacking, and in blue- and violet-sensitive receptors the $\beta$-peak is outside the measured wavelength range, so its amplitude can be estimated only indirectly. Thus, eqns. (4), (5), and (8) should be considered only as approximate.

\section{$\beta$-band absorbance and sensitivity}

In the $\beta$-band region, there is a remarkable variation in the relation between MSP and sensitivity spectra. For several cell types, especially cones, electrophysiological sensitivity in the near-UV has been reported to be about $10 \%$ of maximum, that is, 2 - to 3-fold lower than absorptance. In Fig. 16, the four lower panels exemplify various degrees of discrepancy observed in Al photoreceptors. The two top panels, on the other hand, show that a reasonable agreement is found for some cells even in this range. Among A2 photoreceptors, the red-sensitive cones of carp and "red" rods of Xenopus show a rough agreement between absorptance and sensitivity, while in most other cells, $\beta$-band sensitivity is substantially lower than absorptance (data not shown). It is not clear whether the discrepancy indicates a difference in the quantum yield between the $\alpha$ - and $\beta$-band, or if further improvement of the techniques will bring electrophysiology and MSP into closer agreement.

\section{Meanings of $\lambda_{\max }$}

There is no way of identifying a "true" peak in noisy data without assumptions on the shape of the underlying function. Thus, the "wavelength of maximum absorbance" $\lambda_{\max }$ is always a parameter of fitting data with a certain predetermined template, and, as such, depends of what template is used (cf. Levine \& MacNichol, 1985). For this reason, different authors may give significantly different $\lambda_{\max }$ values for the same visual pigment, and this need not indicate
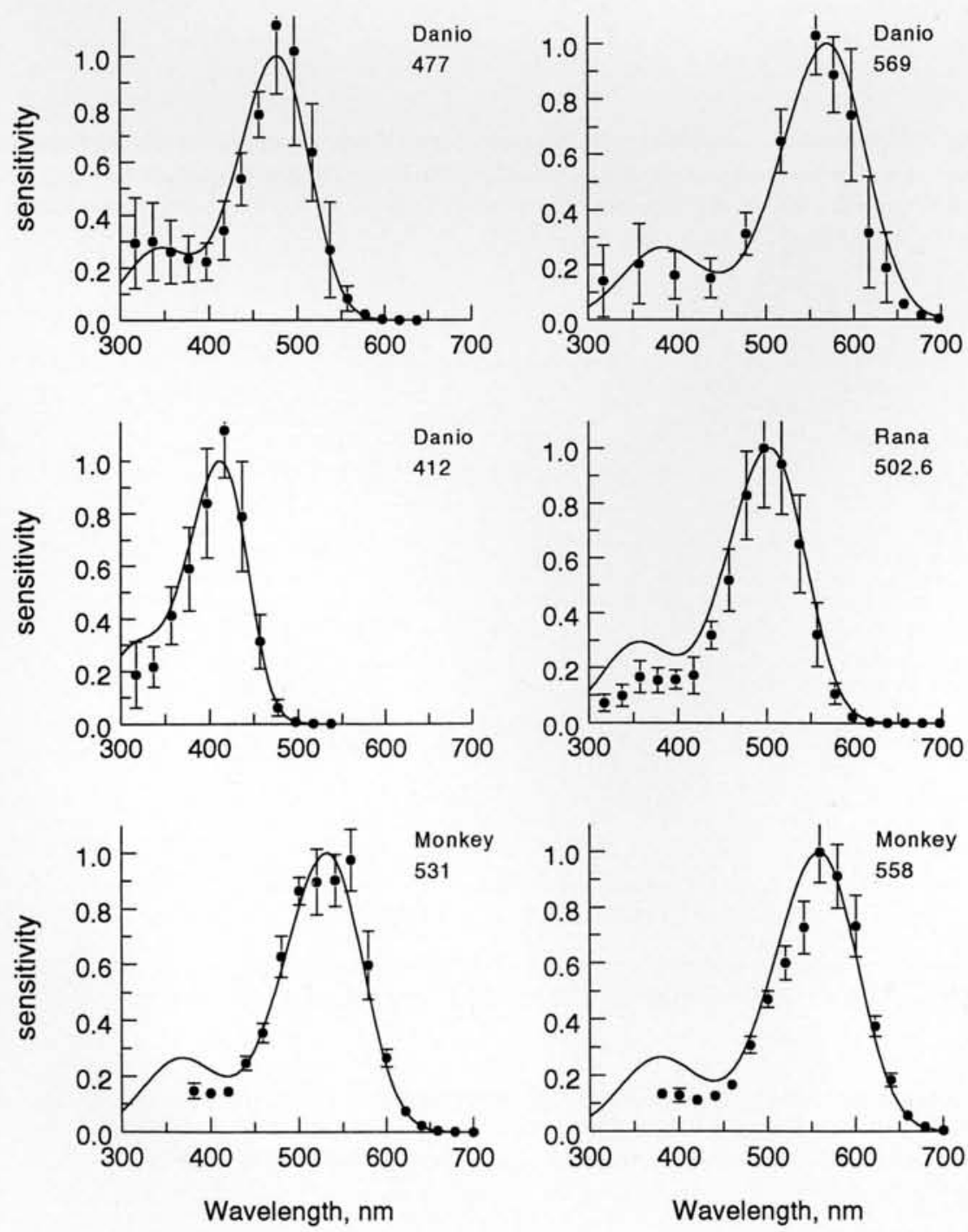

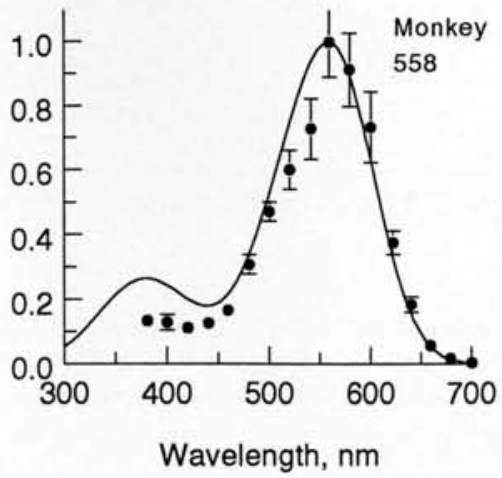

Fig. 16. Comparison of sensitivity and absorptance in the $\beta$-band region of photoreceptors with $\mathrm{Al}$ pigments. Templates generated by eqns, (1)-(5) are compared with electrophysiological data by Palacios et al. (1996, 1998a; Danio and Rana pipiens) and Baylor et al. (1987; macaque). The two top panels show reasonably good agreement between the two properties, while in the bottom panels sensitivity is almost two-fold lower than absorptance in the $\beta$-band. 
any real differences (e.g. genetic polymorphism) or inaccuracy of data. Anyway, high accuracy in knowing the location of a "true" maximum is unimportant as such, since the peak is so broad that curves may be significantly shifted without experimentally measurable changes in absorbance at this spectral location. Similarly, it is generally useless to argue about differences in the $\beta$-band parameters given by different authors. Only the actual curves specified by the parameters can be reasonably compared.

A standardized template allows unambiguous curve-fitting based on the full set of data. If the shape of the visual pigment absorbance curve is indeed uniquely related to its wavelength of maximum absorbance, the parameter $\lambda_{\max }$ will then completely characterize the whole spectrum and can be used for genuine comparisons across species and experimenters. We propose that a valid template for the $\alpha$-band of Al pigments can be based on the Partridge and De Grip (1991) bovine standard, mathematically described by Lamb's (1995) formula equipped with our modified MM transform [eqns. (1) and (2)]. For A2 pigments, Bridges' (1967) porphyropsin data, described by Lamb's formula with other parameters, and equipped with a different modification of the MM transform [eqns. (1) and (6)], can be used. As pointed out before, the analytical form is basically arbitrary, and it is comparatively uninteresting whether more aesthetic expressions may be devised to describe the existing data. The important question is whether new experimental data may force more substantial revisions.

\section{Exceptions from universality}

The general conclusion that it may be possible to describe the absorbance/sensitivity spectra of all vertebrate photoreceptors by the proposed $\mathrm{A} 1$ or $\mathrm{A} 2$ template (or a combination of an A1/A2 pair) is subject to some reservations:

1. For the idea of universality, the most problematic experimental data at present appear to be the (electrophysiological) sensitivity spectra of ground squirrel rods and cones reported by Kraft (1988). These are markedly wider than the bovinebased template, and the reason(s) need to be clarified. The widening is apparently not due to a mixture of different chromophores (Makino et al., 1990), but might well be due, for example, to a co-expression of two opsins with close $\lambda_{\max }$ in the same cell [cf. point (2)].

2. The tail of the spectrum of the Danio UV-cone at $\lambda>400 \mathrm{~nm}$ was found to deviate from any conventional template (Fig. 5). A similar deviation has been found in MSP spectra from gecko UV receptors (Loew et al., 1996). Neither Danio nor gecko cells have yet been tested by partial bleaching for the presence of a mixture of visual pigments, as suggested by spectral-sensitivity measurements in UV-sensitive cones of Ambystoma (Makino \& Dodd, 1996). The shoulder in Danio would be explained by a $3.5 \%$ admixture of the visual pigment of the violet-sensitive cones. Interestingly, no shoulder appears in the spectral-sensitivity data of Palacios et al. (1996), indicating a difference between fish from different sources.

3. Absorbance properties of long-wave cone visual pigments are affected by $\mathrm{pH}$ and anions present in the solution (Fager \& Fager, 1979; Crescitelli, 1980, 1981; Govardovskii et al., 1985; Novitskii et al., 1989; Kleinschmidt \& Hárosi, 1992). Variable mixtures of protonated/unprotonated and $\mathrm{Cl}^{-}$-free/ $\mathrm{Cl}^{-}$-bound forms of the pigment could produce variable spectra that would strongly diverge from any template. The effect is undoubtedly important in experimental conditions. It is not clear, however, whether in the normal physiological state cytoplasmic $\left[\mathrm{H}^{+}\right]$and $\left[\mathrm{Cl}^{-}\right]$levels can be low enough to allow a noticeable concentration of an "unusual" form. Our absorbance spectra of $\mathrm{Cl}^{-}$-sensitive pigments of Danio and geckos give no evidence of such an effect, being accurately fitted by a common template.

4. The shape of the long-wavelength decline (cf. Figs. 14 and 15 ) is not strictly speaking a fixed property of a spectrum, but depends on temperature. Warming increases the probability that long-wavelength photons isomerize rhodopsin in solution (St. George, 1952) and decreases the slope as measured by electrophysiology (Srebro, 1966; Lamb, 1984; Koskelainen et al., 2000). This is qualitatively consistent with the idea of Stiles (1948), further developed by Lewis (1955), predicting that the relative probability for low-energy ("red") photons to produce excitations should increase with increasing availability of thermal energy. The effect is measurable only outside the range accessible to $\operatorname{MSP}(\lambda>640 \mathrm{~nm})$, it is weaker for A2 than A1 pigments, and its magnitude is not simply related to $\lambda_{\max }$. For example, in both the rhodopsin rods and red-sensitive cones of frog warming from 5 to $25^{\circ} \mathrm{C}$ decreases the final slope of $\log$ sensitivity versus $1 / \lambda$ by $c a$. $10 \%$ (Koskelainen et al., 2000). Thus, this effect cannot be incorporated into a template quite so simply as suggested by Mooij and van den Berg (1983).

At this stage, none of these fundamentally challenges our argument. If (1) and (2) are due to pigment mixtures, they are trivial from the viewpoint of pigment templates. The importance of (3) in physiological conditions should be evaluated. The effects of (4) are subtle (revealed only by careful electrophysiological measurements) and can in principle be taken into account in a refined template.

\section{Conclusions}

A template based on the bovine rhodopsin absorbance data of Partridge and De Grip (1991), of the analytical form proposed by Lamb (1995), and incorporating a modification of the MansfieldMacNichol transform, was found to provide a good fit to $\alpha$-band absorbance/sensitivity in Al rods and cones, covering the whole range of $\lambda_{\max }$ and representing ten species of mammals, reptiles, amphibians, and fish. Analogous modification of a Lamb-type template based on the carp porphyropsin absorbance data of Bridges (1967) provided a good fit to $\alpha$-band absorbance/sensitivity in A2 rods and cones of six fish and amphibian species. Together with approximate expressions proposed for the $\beta$-band, our templates provide a complete description of absorbance and spectral-sensitivity curves in the wavelength range $\lambda>330 \mathrm{~nm}$.

\section{Acknowledgments}

The authors are grateful to Edward MacNichol, Jr., Gregor Jones, and Felix Gribakin for comments on the manuscript; and to Ellis Loew for permission to use unpublished data on turtle visual pigments. Vadim Maximov helped us with refinement of the A2-pigment template. The infrared viewers in St. Petersburg are a gift of the late Roy Steinberg (UCSF), and the Helsinki photomultiplier is a gift of Ellis Loew (Cornell University). Clint Makino (MEEI) kindly provided his tabulated data on spectral sensitivity of salamander cones. This work formed part of joint project No 22a of the Russian Academy of Sciences and the Academy of Finland. It was further supported by grants from the Russian Foundation for Basic Research (\# 9304-7582 and 99-04-49952) and from the Academy of Finland (\#36154), by 
the Sigrid Jusélius Foundation, and by the Finnish Graduate School of Neuroscience. A preliminary version of the paper was presented at the 6 th Congress of the European Society for Photobiology in 1995.

\section{References}

BARLow, H.B. (1982). What causes trichromacy? A theoretical analysis using comb-filtered spectra. Vision Research 22, 635-643.

Baylor, D.A., NunN, B.J. \& Schnapf, J.L. (1984). The photocurrent, noise, and spectral sensitivity of rods of the monkey Macaca fascicularis. Journal of Physiology 357, 575-607.

BAYLOR, D.A., NUNN, B.J. \& SCHNAPF, J.L. (1987). Spectral sensitivity of cones of the monkey, Macaca fascicularis. Journal of Physiology 390, $145-160$.

BowmAKER, J.K. (1972). Kundt's rule: The spectral absorbance of visual pigments in situ and in solution. Vision Research 12, 529-548.

BOWMAKER, J.K. (1973). Spectral sensitivity and visual pigment absorbance. Vision Research 13, 783-792.

Bowmaker, J.K., Loew, E.R. \& Liebman, P.A. (1975). Variation in the $\lambda_{\max }$ of rhodopsin from individual frogs. Vision Research 15, 997-1003.

BRIDGES, D. (1967). Spectroscopic properties of porphyropsins. Vision Research 7, 349-369.

Cornwall, M.C., MaCNichOl, E.F., JR. \& Fein, A. (1984). Absorbance and spectral sensitivity measurements of rod photoreceptors of the tiger salamander, Ambystoma tigrinum. Vision Research 24, 1561-1569.

Crescitelli, F. (1958). The natural history of visual pigments. Annals of the New York Academy of Science 74, 230-255.

Crescitelli, F. (1980). The gecko visual pigments: The nitrate effects. Vision Research 20, 937-945.

Crescitelli, F. (1981). The gecko visual pigment: A pH indicator with a salt effect. Journal of Physiology 321, 385-399.

Crescitelli, F., Dartnall, H.J.A. \& Loew, E.R. (1977).The gecko visual pigments: A microspectrophotometric study. Journal of Physiology 268, 559-573.

DARTNALL, H.J.A. (1953). The interpretation of spectral sensitivity curves, British Medical Bulletin 9, 24-30.

DaRTNAll, H.J.A. (1961). Visual pigments before and after extraction from visual cells. Proceedings of the Royal Society B 154, 250-266.

Dartnall, H.J.A. \& Lythgoe, J.N. (1965). The spectral clustering of visual pigments. Vision Research 5, 81-100.

DawIs, S.M. (1981). Polynomial expression of pigment nomograms. Vision Research 21, 1427-1430.

DEnton, E.J. (1959). The contribution of oriented photosensitive and other molecules to the absorption of whole retina. Proceedings of the Royal Society B 150, 78-94.

DONNER, K.O. \& REUTER, T. (1976). Visual pigments and photoreceptor function. In Frog Neurobiology, ed. Llinás, R. \& PRECHT, W., pp. 251277. Berlin-Heidelberg-New York: Springer.

DonNer, K., Firsov, M.L. \& GovardovskiI, V.I. (1990). The frequency of isomerization-like"dark" events in rhodopsin and porphyropsin rods of the bull-frog retina. Journal of Physiology 428, 673-692.

EBREY, T.G. \& HoNIG, B. (1977). New wavelength-dependent visual pigment nomogram. Vision Research 17, 147-151.

FAGER, L.Y. \& FAGER, R.S. (1979). Halide control of color of the chicken cone pigment iodopsin. Experimental Eye Research 29, 401-408.

GovardovSKII, V.I., ZAK, P.P. \& NovITSKII, I.YU. (1985). Ionochromic changes of absorption spectra of gecko photoreceptors. Biofizika $\mathbf{3 0}$, 292-296 (in Russian).

Greenberg, A.D., Honig, B. \& Ebrey, T.G. (1975). Wavelength dependence of the bandwidths of visual pigment spectra. Nature 257 , 823-824.

Griffin, D.R., HubBard, R. \& WALD, G. (1947). The sensitivity of the human eye to infra-red radiation. Journal of the Optical Society of America 37, 546-554.

HÁrosi, F.I. (1975a). Microspectrophotometry: The technique and some of its pitfalls. In Vision in Fishes. New Approaches in Research, ed. Al., M.A., pp. 43-54. New York and London: Plenum.

HÁrosi, F.I. (1975b). Absorption spectra and dichroism of some amphibian photoreceptors. Journal of General Physiology 66, 357-382.

HÁrosi, F.I. (1976). Spectral relations of cone visual pigments in goldfish. Journal of General Physiology 68, 65-80.

Hárosi, F.I. (1994). An analysis of two spectral properties of vertebrate visual pigments. Vision Research 34, 1359-1367.

HÁrosi, F.I. \& MACNichOL, E.F., JR. (1974a). Visual pigments of goldfish cones. Journal of General Physiology 63, 279-304.
Hárosi, F.I. \& MACNichOL, E.F., JR. (1974b). Dichroic microspectrophotometer: A computer-assisted, rapid, wavelength-scanning photometer for measuring linear dichroism in single cells. Journal of the Optical Society of America 64, 903-918.

Johnson, R.L., Grant, K.B., Zankel, T.C., Boehm, M.F., Merbs, S.L., NATHANS, J. \& NAKANISHI, K. (1993). Cloning and expression of goldfish opsin sequences. Biochemistry 32, 208-214.

KleinschmidT, J. \& Hárosi, F.I. (1992). Anion sensitivity and spectral tuning of cone visual pigments in situ. Proceedings of the National Academy of Sciences of the U.S.A. 89, 9181-9185.

KnOWLES, A. \& DarTnal., H.J.A. (1977). The photobiology of vision. In The Eye, Vol. 2B, ed. Davson, H., pp. 53-101. London-New York: Academic Press.

Koskelainen, A., Ala-Laurila, P., Fyhrquist, N. \& Donner, K. (2000). Measurement on thermal contribution to photoreceptor sensitivity. $\mathrm{Na}$ ture 403, 220-223.

KRAFT, T.W. (1988). Photocurrent of cone photoreceptors of the goldenmantled ground squirrel. Journal of Physiology 404, 199-213.

Kraft, T.W., SChNEEWEIS, D.M. \& SCHNAPF, J.L. (1993). Visual transduction in human rod photoreceptors. Journal of Physiology 464. $747-765$.

LAMB, T.D. (1984). Effects of temperature changes on toad rod photocurrents. Journal of Physiology 346, 557-578.

LAMB, T.D. (1995). Photoreceptor spectral sensitivities: Common shape in the long-wavelength region. Vision Research 35, 3083-3091.

LEVINE, J.S. \& MACNichOL, E.F., JR. (1985). Microspectrophotometry of primate photoreceptors: Art, artifact, and analysis. In The Visual System, ed. Fein, A. \& Levine, J.S., pp. 73-87. New York: Alan R. Liss.

LEWIS, P.R. (1955). A theoretical interpretation of spectral sensitivity curves at long wavelengths. Journal of Physiology 130, 45-52.

LieBMAN, P.A. (1972). Microspectrophotometry of photoreceptors. In Handbook of Sensory Physiology, VII/l. Photochemistry of Vision, ed. Dartnall, H.J.A., pp. 481-528. Berlin-Heidelberg-New York: Springer.

Liebman, P.A. \& Entine, G. (1968). Visual pigments of frog and tadpole (Rana pipiens). Vision Research 8, 761-775.

Loew, E.R., GovardovskiI, V.I., Rölich, P. \& Szél, Á. (1996). Microspectrophotometric and immunocytochemical identification of ultraviolet photoreceptors in geckos. Visual Neuroscience 13, 247256.

MACNichol, E.F., JR. (1986). A unifying presentation of photopigment spectra. Vision Research 26, 1543-1556.

Makino, C.L., Kraft, T.W., Mathies, R.A., Lugtenburg, J., Miley, M.E., vaN DER STEEN, R. \& BAYLOR, D.A. (1990). Effects of modified chromophores on the spectral sensitivity of salamander, squirrel, and macaque cones. Journal of Physiology 424, 545-560.

MAKINO, C.L., TAYLOR, W.R. \& BAYLOR, D.A. (1991). Rapid charge movement and photosensitivity of visual pigments in salamander rods and cones. Journal of Physiology 442, 761-780.

MANSFIELD, R.J.W. (1985). Primate photopigments and cone mechanisms. In The Visual System, ed. Fein, A. \& Levine, J.S., pp. 89-106. New York: Liss.

Mansfield, R.J.W., Levine, J.S., Lipetz, L.E., Oleszko-Szuts, S. \& MACNichOL, E.F., JR. (1986). Vertebrate visual pigments: Canonical form of the absorbance spectra $\alpha$-band. Investigative Ophthalmology and Visual Science 27, 193.

MARKS, W.B. (1965). Visual pigments of single goldfish cones. Journal of Physiology 178, 14-32.

Maximov, V.V. (1988). Approximation of visual pigment absorbance spectra. Sensornye Sistemy 2, 3-9 (in Russian).

MetZleR, D.E. \& HARRIS, C.M. (1978). Shapes of spectral bands of visual pigments. Vision Research 18, 1417-1420.

MOOI, J.E.M. \& VAN DEN BERG, T.J.T.P. (1983). The spectral shape of A2 visual pigments. Vision Research 23, 701-705.

MUNTZ, W.R.A. \& REUTER, T. (1966). Visual pigments and spectral sensitivity in Rana temporaria and other European tadpoles. Vision Research 6, 601-618.

Munz, F.W. \& Schwanzara, S.A. (1967). A nomogram for retinene $2^{-}$ based visual pigments. Vision Research 7, 111-120.

NAKA, K.I. \& Rushton, W.A.H. (1966). An attempt to analyse colour vision by electrophysiology. Journal of Physiology 185, 556-586.

Novitskil, I.YU., ZAK, P.P. \& OstrovskII, M.A. (1989). The effect of anions on the spectral properties of iodopsin in native cones of the frog retina (a microspectrophotometric study). Bioorganicheskaya Khimiya 15, 1037-1043 (in Russian). 
Palacios, A.G., Goldsmith, T.H. \& Bernard, G.D. (1996). Sensitivity of cones from a cyprinid fish (Danio aequipinnatus) to ultraviolet and visible light. Visual Neuroscience 13, 411-421.

Palacios, A.G., Srivastava, R. \& Goldsmith, T.H. (1998a). Spectral and polarization sensitivity of photocurrents of amphibian rods in the visible and ultraviolet. Visual Neuroscience 15, 319-331.

Palacios, A., Varela, F.J., Srivastava, R. \& Goldsmith, T.H. (1998b). Spectral sensitivity of cones in the goldfish, Carassius auratus. Vision Research 38, 2135-2146.

PARTRIDGe, J.C. \& DE GRIP, W.J. (1991). A new template for rhodopsin (vitamin A1 based) visual pigments. Vision Research 31, 619-630.

Partridge, J.C., Speare, P., Shand, J., Muntz, W.R.A. \& Williams, D.McB. (1992). Microspectrophotometric determinations of rod visual pigments in some adult and larval Australian amphibians. Visual Neuroscience 9, 137-142.

REUTER, T. (1969). Visual pigments and ganglion cell activity in the retinae of tadpoles and adult frogs (Rana temporaria L.). Acta Zoologica Fennica 122, 1-64.

REUTER, T.E., WhItE, R.H. \& WALD, G. (1971). Rhodopsin and porphyropsin fields in the adult bullfrog retina. Journal of General Physiology 58, 351-371.

SaARikoski, J., RuUsuvuori, E., Koskelainen, A. \& Donner, K. (1997). Regulation of intracellular $\mathrm{pH}$ in salamander retinal rods. Journal of Physiology 498, 61-72.

SREBRo, R. (1966). A thermal component of excitation in the lateral eye of Limulus. Journal of Physiology 187, 417-425.

Stavenga, D.G., Smits, R.P. \& Hoenders, B.J. (1993). Simple exponential functions describing the absorbance bands of visual pigment spectra. Vision Research 33, 1011-1017.

ST. GEORGE, R.C.C. (1952). The interplay of light and heat in bleaching rhodopsin. Journal of General Physiology 35, 495-517.

STILES, W.S. (1948). The physical interpretation of the spectral sensitivity curve of the eye. In Transactions of the Optical Convention of the Worshipful Company of Spectacle Makers, pp. 97-107. London: Spectacle Makers' Co.

Tsin, A.T.C. \& BEATTY, D.D. (1980). Visual pigments and vitamins A in the adult bullfrog. Experimental Eye Research 30, 143-153.

Tsin, A.T.C., Liebman, P.A., Beatty, D.D. \& Drzymala, R. (1981). Rod and cone visual pigments in the goldfish. Vision Research 21, 943-946.

WALD, G. (1946). The metamorphosis of visual systems in amphibia. Biological Bulletin 91, 239-240.

Whitmore, A.V. \& Bowmaker, J.K. (1989). Seasonal variation in cone sensitivity and short-wave absorbing visual pigments in the rudd Scardinius erythrophthalmus. Journal of Comparative Physiology A 166, 103-115.

\section{Appendix 1}

\section{Correction for bleaching}

The purpose of the procedure is to allow the use of a fairly high measuring light flux (to achieve a high SNR) while still minimizing spectral artifacts due to bleaching of visual pigment. Basically, bleaching during the spectral scan has a triple effect on the recorded spectrum (Liebman, 1972; Hárosi \& MacNichol, 1974b). First (and least harmful), the maximum absorbance is decreased. Second, the spectrum is skewed due to progressive absorbance loss during the scan. Third, it is distorted due to the accumulation of bleaching products. The idea of correction for bleaching was introduced by Marks (1965), but his computational approach had to rely on rather uncertain assumptions about the bleach at different wavelengths and the time-dependent behavior of bleaching products. Therefore, we devised a simple correction procedure that relies on measuring the effects of relatively small bleaches.

The bare idea looks as follows. Assume that, owing to bleaching during a scan with beam intensity $I_{1}$ ("unit" measuring intensity), the recorded absorbance spectrum $A_{1}(\lambda)$ diverges from the genuine "dark" spectrum $A_{d}(\lambda)$ by the component $\mathrm{a}(\lambda)$, that is,

$$
A_{1}(\lambda)=A_{d}(\lambda)+a(\lambda)
$$

If the same cell were recorded with a two times higher beam intensity $I_{2}=2 I_{1}$ ("double" measuring intensity), and $a(\lambda) / A_{d}(\lambda) \ll 1$, the distor- tion by bleaching is expected to be approximately $2 a(\lambda)$, that is,

$$
A_{2}(\lambda)=A_{d}(\lambda)+2 a(\lambda)
$$

Thus, the correction factor, $a(\lambda)$, is obtained as

$$
a(\lambda)=A_{2}(\lambda)-A_{1}(\lambda),
$$

and the dark spectrum as

$$
A_{d}(\lambda)=A_{1}(\lambda)-a(\lambda) \text {, }
$$

or

$$
A_{d}=A_{2}(\lambda)-2 a(\lambda)
$$

In reality, the effect of bleaching tends to saturate with increasing light intensity, so eqn. (A3) is an underestimate of the correction factor. The extra error should be approximately proportional to $\left[a\left(\lambda_{\max }\right) / A_{d}\left(\lambda_{\max }\right)\right]^{2}$.

In practice, we selected $I_{1}$ so that the maximum density decrease after a single scan was $2-3 \%$, and $5-6 \%$ with double intensity. The transient local bleach during the scan, until the diffusion equilibrium is established, may be substantially larger, probably close to $10 \%$. The correction factor would then be underestimated by less than 0.01 , which we can neglect at present.

The actual procedure is illustrated in Fig. 17. In principle, the recordings with "unit" and "double" intensity might be done from the same OS, for example, from the proximal and distal end, respectively. Usually it is more convenient, however, to make alternating recordings with two beam intensities from different OSs of approximately the same size. Both sets of data are averaged and the zero line, as defined by linear regression on the last 100 points (between 650 and $750 \mathrm{~nm}$ ), subtracted. Then the curves should be normalized to remove a possible difference between the two scanned rod populations. The strict way to do this is to normalize the $I_{1}$ record to 1 at its maximum, and the $I_{2}$ record to $\left[1+a\left(\lambda_{\max }\right) / A\left(\lambda_{\max }\right)\right]$ at the same wavelength (as has been done in Fig. 17A). It is easy to show that, with our system of recording, whereby two spectral scans in opposite directions are averaged, $a\left(\lambda_{\max }\right)=\Delta A\left(\lambda_{\max }\right) / 2$, where $\Delta A\left(\lambda_{\max }\right)<0$ is the density loss between the first and second scan of the same intensity. (This presupposes that the bleach is small and no bleaching product is formed at $\lambda_{\max }$ ). The correction factor can then be obtained as the difference between the two curves.

In most cases, however, we did not care to obtain an accurate estimate of the degree of bleaching. Since $a\left(\lambda_{\max }\right) / A\left(\lambda_{\max }\right) \ll 1$ (e.g. 0.02 in Fig. 17A), the two curves can be normalized to 1 . This will result in a small overestimate of the short-wave correction that will partly compensate for the underestimate due to the assumption of negligible bleaching in eqn. (A3). To avoid extra noise, the correction curve is heavily low-pass filtered (thick smooth line close to zero in Fig. 17A). The correction is made in both the "unit" and the "double" intensity spectrum [eqns. (A4) and (A5)] and further noise reduction is achieved by averaging the two corrected spectra, which now differ in high-frequency noise only (Fig. 17B). The resulting curve shows an excellent fit to the bovine template MM transformed to $502.6 \mathrm{~nm}$ (dots).

\section{Appendix 2}

\section{Control of light scatter and background absorbance in the $\beta$-band range}

The spectrum in the short-wave range is especially susceptible to distortion by light scatter and possible absorbing material other than visual pigments and their bleaching products. To obtain the $\beta$-band absorbance of the native visual pigment, it is essential that these effects be eliminated or at least estimated as well as possible. 

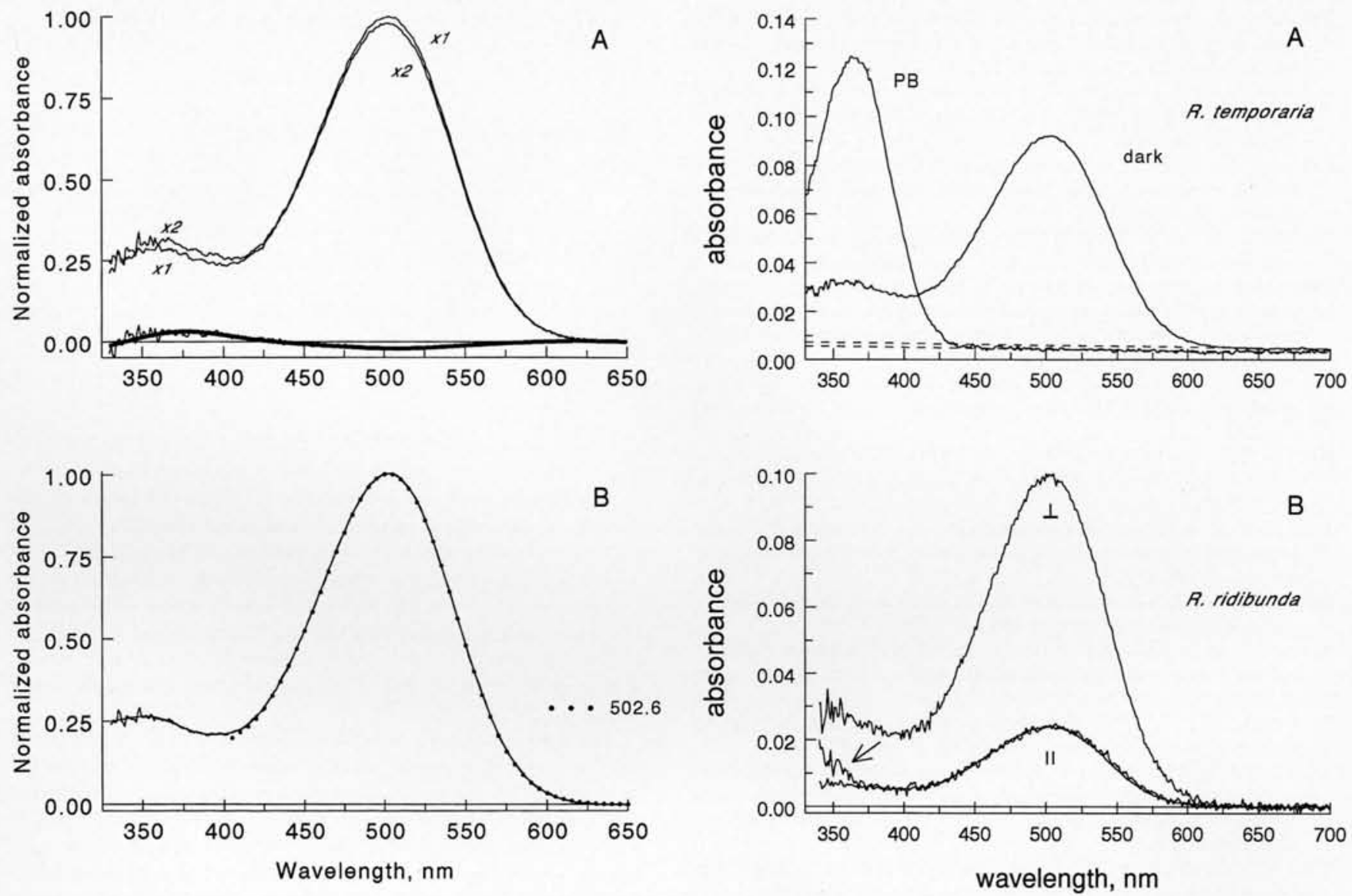

Fig. 17. The procedure of correction for bleaching. Data from rhodopsin rods of Rana temporaria. (A) The spectrum recorded at "unit" intensity is marked $x l$ (average of $27 \mathrm{OS}$ ), and the spectrum recorded at "double" intensity is marked $x 2$ (average of $10 \mathrm{OS}$ ). The zero line has been drawn and subtracted as in Fig. 1, and the spectra have been normalized as described in Appendix 1. The thin noisy curve near zero absorbance is the correction factor, that is, the difference between the $x 2$ and $x l$ spectra. The smooth bold line is the same correction factor after Fourier filtering with 12 harmonics retained. (B) The thin noisy curve is the corrected absorbance spectrum obtained as described in the text. The smooth curve is the same spectrum after Fourier filtering with 35 harmonics retained. The dots give the bovine template MM transformed to $\lambda_{\max }=502.6 \mathrm{~nm}$.

Fig. 18A shows that light scatter is insignificant in our recordings from amphibian rods. A straight regression line fitted to points of near-zero absorbance (between 630 and $750 \mathrm{~nm}$ in the record marked "dark") is almost horizontal, with slope less than $10^{-5} \mathrm{~nm}^{-1}$. The much wider nearzero absorbance range $(450-750 \mathrm{~nm})$ available in a hydroxylamine postbleach spectrum (marked PB) yielded virtually the same slope. This justifies extrapolation into the $\beta$-band region of a zero line obtained by a linear fit to the long-wave tail of the spectrum. Recordings from the violet- and UV-sensitive cones in Danio also provide a long stretch of near-zero absorbance (from 450 or 500 to $750 \mathrm{~nm}$ ), which is well approximated by a straight line and can be safely extended into the $\beta$-band region (not shown).

The possible interference of extra light-absorbing material (and, at the same time, the correctness of the zero-line subtraction) was checked by comparing absorbances at two orientations of the $e$-vector of the measuring beam, across $\left(A_{\perp}\right)$ and along $\left(A_{\|}\right)$the OS axis (Fig. 18B). These recordings were done at low light intensity, resulting in less than $1 \%$ final bleaching. The $A_{\|}$spectrum was recorded first, so bleaching during the first scan was negligible due to low absorbance. The dichroic ratio $A_{\perp} / A_{\|}$(cf. Denton, 1959; Hárosi \& MacNichol, 1974b; Hárosi, 1975b) in this sample of rods

Fig. 18. Assessing the reliability of absorbance data in the spectral region of the $\beta$-band. (A) The validity of the zero-line subtraction (data from rhodopsin rods of Rana temporaria). The dark-adapted spectrum is the average of $18 \mathrm{OS}$, and the postbleach spectrum is the average of six OS; $20 \mathrm{mM}$ hydroxylamine added to the sample. Neither spectrum has been filtered or bleach corrected. The zero lines (dashed) are extrapolations of least-square fits to the stretch from 630 to $750 \mathrm{~nm}$ (dark), and from 450 to $750 \mathrm{~nm}$ (postbleach, denoted PB). The dark spectrum has been shifted upwards for clarity; in reality, the two spectra coincide at the longest wavelengths. The fact that the zero lines determined from both spectra are virtually horizontal (slopes $7.53 \times 10^{-6} \mathrm{~nm}^{-1}$ and $7.16 \times 10^{-6} \mathrm{~nm}^{-1}$ ) and coincide justifies extrapolating them into the $\beta$-band region. (B) Absence of significant absorbance other than visual pigment and its bleaching products: comparison of OS absorbance recorded with two $e$-vector orientations, along $(\|)$ and across $(\perp)$ the OS axis. Data from rhodopsin rods of Rana ridibunda. Both spectra are averages from $16 \mathrm{OS}$, not filtered nor bleach corrected. The low-noise line superimposed on the $\|$-spectrum is the $\perp$-spectrum multiplied by 0.24 . The curves are virtually coincident down to $370 \mathrm{~nm}$. The arrow indicates extra short-wave absorbance of a substance (possibly retinol) oriented predominantly along the OS axis.

was 4.16. When scaled to the same $\alpha$-peak, the $A_{\perp}$ and $A_{\|}$spectra coincide down to at least $375 \mathrm{~nm}$. At even shorter wavelengths, the $A_{\|}$spectrum exhibits a steep increase in absorbance (arrow in Fig. 18B) manifesting the presence of some extra substance with maximum absorbance below $350 \mathrm{~nm}$ and predominant orientation across the disk plane. A similar absorbance increase has been noticed earlier (Hárosi \& MacNichol, 1974b; Hárosi,1975b). Free retinol is one candidate, having the right $\lambda_{\max }$ and orientation in the membrane (Denton, 1959; Hárosi \& MacNichol, 1974b; Hárosi, 1975b). This short-wave absorbance increase varied between retinas. Fig. 18B shows one of the most prominent examples. Its contribution to the $A_{\perp}$ spectrum is small and could be neglected. 